\title{
Hypergraphic LP Relaxations for Steiner Trees
}

\author{
Deeparnab Chakrabarty Jochen Könemann David Pritchard \\ University of Waterloo *
}

\begin{abstract}
We investigate hypergraphic LP relaxations for the Steiner tree problem, primarily the partition LP relaxation introduced by Könemann et al. [Math. Programming, 2009]. Specifically, we are interested in proving upper bounds on the integrality gap of this LP, and studying its relation to other linear relaxations. Our results are the following.

Structural results: We extend the technique of uncrossing, usually applied to families of sets, to families of partitions. As a consequence we show that any basic feasible solution to the partition LP formulation has sparse support. Although the number of variables could be exponential, the number of positive variables is at most the number of terminals.

Relations with other relaxations: We show the equivalence of the partition LP relaxation with other known hypergraphic relaxations. We also show that these hypergraphic relaxations are equivalent to the well studied bidirected cut relaxation, if the instance is quasibipartite.

Integrality gap upper bounds: We show an upper bound of $\sqrt{3} \doteq 1.729$ on the integrality gap of these hypergraph relaxations in general graphs. In the special case of uniformly quasibipartite instances, we show an improved upper bound of $73 / 60 \doteq 1.216$. By our equivalence theorem, the latter result implies an improved upper bound for the bidirected cut relaxation as well.
\end{abstract}

\section{Introduction}

In the Steiner tree problem, we are given an undirected graph $G=(V, E)$, non-negative costs $c_{e}$ for all edges $e \in E$, and a set of terminal vertices $R \subseteq V$. The goal is to find a minimum-cost tree $T$ spanning $R$, and possibly some Steiner vertices from $V \backslash R$. We can assume that the graph is complete and that the costs induce a metric. The problem takes a central place in the theory of combinatorial optimization and has numerous practical applications. Since the Steiner tree problem is NP-hard ${ }^{1}$ we are interested in approximation algorithms for it. The best published approximation algorithm for the Steiner tree problem is due to Robins and Zelikovsky [29], which for any fixed $\epsilon>0$, achieves a performance ratio of $1+\frac{\ln 3}{2}+\epsilon \doteq 1.55$ in polynomial time; an improvement is currently in press [3], see also Remark 1.1.

In this paper, we study linear programming (LP) relaxations for the Steiner tree problem, and their properties. Numerous such formulations are known (e.g., see [1, 7, 8, 10, 11, 18, 24, 25, 35, 36]), and their study has led to impressive running time improvements for integer programming based methods. Despite the significant body of work in this area, none of the known relaxations is known

\footnotetext{
*Supported by NSERC grant no. 288340 and by an Early Research Award. Email: (deepc, jochen, dagpritc @uwaterloo.ca)

${ }^{1}$ Chlebík and Chlebíková show that no $(96 / 95-\epsilon)$-approximation algorithm can exist for any positive $\epsilon$ unless $\mathrm{P}=\mathrm{NP}$ [5].
} 
to exhibit an integrality gap provably smaller ${ }^{2}$ than 2 . The integrality gap of a relaxation is the maximum ratio of the cost of integral and fractional optima, over all instances. It is commonly regarded as a measure of strength of a formulation. One of the contributions of this paper are improved bounds on the integrality gap for a number of Steiner tree LP relaxations.

A Steiner tree relaxation of particular interest is the bidirected cut relaxation $[11,36]$ (precise definitions will follow in Section 1.2). This relaxation has a flow formulation using $O(|E||R|)$ variables and constraints, which is much more compact than the other relaxations we study. Also, it is also widely believed to have an integrality gap significantly smaller than 2 (e.g., see [4, 28, 34]). The largest lower bound on the integrality gap known is 8/7 (by Martin Skutella, reported in [23]), and Chakrabarty et al. [4] prove an upper bound of $4 / 3$ in so called quasi-bipartite instances (where Steiner vertices form an independent set).

Another class of formulations are the so called hypergraphic LP relaxations for the Steiner tree problem. These relaxations are inspired by the observation that the minimum Steiner tree problem can be encoded as a minimum cost hyper-spanning tree (see Section 1.2.2) of a certain hypergraph on the terminals. They are known to be stronger than the bidirected cut relaxation [26], and it is therefore natural to try to use them to get better approximation algorithms, by drawing on the large corpus of known LP techniques. In this paper, we focus on one hypergraphic LP in particular: the partition LP of Könemann et al. [23].

\subsection{Our Results and Techniques}

There are three classes of results in this paper: structural results, equivalence results, and integrality gap upper bounds.

Structural results, Section 2: We extend the powerful technique of uncrossing, traditionally applied to families of sets, to families of partitions. Set uncrossing has been very successful in obtaining exact and approximate algorithms for a variety of problems (for instance, [13, 21, 31]). Using partition uncrossing, we show that any basic feasible solution to the partition LP has at most $(|R|-1)$ positive variables (even though it can have an exponentially large number of variables and constraints).

Equivalence results, Section 3: In addition to the partition LP, two other hypergraphic LPs have been studied before: one based on subtour elimination due to Warme [35], and a directed hypergraph relaxation of Polzin and Vahdati Daneshmand [26]; these two are known to be equivalent [26]. We prove that in fact all three hypergraphic relaxations are equivalent (that is, they have the same objective value for any Steiner tree instance). We give two proofs (for completeness and to demonstrate our new techniques), one showing the equivalence of the partition LP and the subtour LP via partition uncrossing, and one showing the equivalence of the partition LP to the directed LP via hypergraph orientation results of Frank et al. [14].

We also show that, on quasibipartite instances, the hypergraphic and the bidirected cut LP relaxations are equivalent. We find this surprising for the following reasons. Firstly, some instances are known where the hypergraph relaxations is strictly stronger than the bidirected cut relaxation [26]. Secondly, the bidirected cut relaxations seems to resist uncrossing techniques; e.g. even in quasi-bipartite graphs extreme points for bidirected cut can have as many as $\Omega\left(|V|^{2}\right)$ positive variables [27, Sec. 4.9]. Thirdly, the known approaches to exploiting the bidirected cut relaxation (mostly primal-dual and local search algorithms [28, 4]) are very different from the combinatorial hypergraphic algorithms for the Steiner tree problem (almost all of them employ greedy strategies).

\footnotetext{
${ }^{2}$ Achieving an integrality gap of 2 is relatively easy for most relaxations by showing that the minimum spanning tree restricted on the terminals is within a factor 2 of the LP.
} 
In short, there is no qualitative similarity to suggest why the two relaxations should be equivalent! We believe a better understanding of the bidirected cut relaxation is important because it is central in theory and practical for implementation.

Improved integrality gap upper bounds, Section 4: For uniformly quasibipartite instances (quasibipartite instances where for each Steiner vertex, all incident edges have the same cost), we show that the integrality gap of the hypergraphic LP relaxations is upper bounded by $73 / 60 \doteq 1.216$. Our proof uses the approximation algorithm of Gröpl et al. [20] which achieves the same ratio with respect to the (integral) optimum. We show, via a simple dual fitting argument, that this ratio is also valid with respect to the LP value. To the best of our knowledge this is the only nontrivial class of instances where the best currently known approximation ratio and integrality gap upper bound are the same.

For general graphs, we give simple upper bounds of $2 \sqrt{2}-1 \doteq 1.83$ and $\sqrt{3} \doteq 1.729$ on the integrality gap of the hypergraph relaxation. Call a graph gainless if the minimum spanning tree of the terminals is the optimal Steiner tree. To obtain these integrality gap upper bounds, we use the following key property of the hypergraphic relaxation which was implicit in [23]: on gainless instances (instances where the optimum terminal spanning tree is the optimal Steiner tree), the LP value equals the minimum spanning tree and the integrality gap is 1 . Such a theorem was known for quasibipartite instances and the bidirected cut relaxation (implicitly in [28], explicitly in [4]); we extend techniques of [4] to obtain improved integrality gaps on all instances.

Remark 1.1. The recent independent work of Byrka et al. [3], which gives an improved approximation for Steiner trees in general graphs, also shows an integrality gap bound of 1.55 on the hypergraphic directed cut LP. This is stronger than our integrality gap bounds and was obtained prior to the completion of our paper; yet we include our bounds because they are obtained using fairly different methods which might be of independent interest in certain settings.

The proof in [3] can be easily modified to show an integrality gap upper bound of 1.28 in quasibi-

partite instances. Then using our equivalence result, we get an integrality gap upper bound of 1.28 for the bidirected cut relaxation on quasibipartite instances, improving the previous best of 4/3.

\subsection{Bidirected Cut and Hypergraphic Relaxations}

\subsubsection{The Bidirected Cut Relaxation}

The first bidirected LP was given by Edmonds [11] as an exact formulation for the spanning tree problem. Wong [36] later extended this to obtain the bidirected cut relaxation for the Steiner tree problem, and gave a dual ascent heuristic based on the relaxation. For this relaxation, introduce two $\operatorname{arcs}(u, v)$ and $(v, u)$ for each edge $u v \in E$, and let both of their costs be $c_{u v}$. Fix an arbitrary terminal $r \in R$ as the root. Call a subset $U \subseteq V$ valid if it contains a terminal but not the root, and let $\operatorname{valid}(V)$ be the family of all valid sets. Clearly, the in-tree rooted at $r$ (the directed tree with all vertices but the root having out-degree exactly 1 ) of a Steiner tree $T$ must have at least one arc with tail in $U$ and head outside $U$, for all valid $U$. This leads to the bidirected cut relaxation $(\mathcal{B})$ (shown in Figure 1 on page 4 with dual) which has a variable for each arc $a \in A$, and a constraint for every valid set $U$. Here and later, $\delta^{\text {out }}(U)$ denotes the set of $\operatorname{arcs}$ in $A$ whose tail is in $U$ and whose head lies in $V \backslash U$. When there are no Steiner vertices, Edmonds' work [11] implies this relaxation is exact.

Goemans \& Myung [18] made significant progress in understanding the LP, by showing that the bidirected cut LP has the same value independent of which terminal is chosen as the root, and by showing that a whole "catalogue" of very different-looking LPs also has the same value; later Goemans [17] showed that if the graph is series-parallel, the relaxation is exact. Rajagopalan and 


$$
\begin{aligned}
& \min \sum_{a \in A} c_{a} x_{a}: \quad x \in \mathbf{R}_{\geq 0}^{A} \\
& \sum_{a \in \delta^{\text {out }}(U)} x_{a} \geq 1, \quad \forall U \in \operatorname{valid}(V)
\end{aligned}
$$

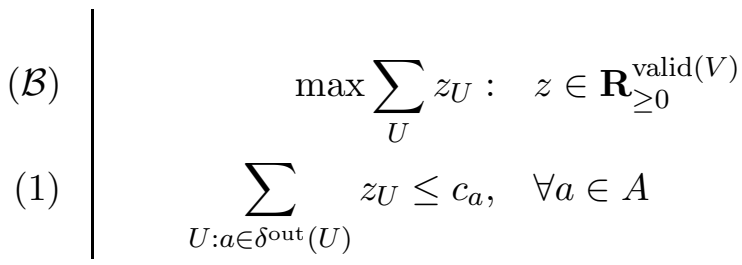

Figure 1: The bidirected cut relaxation $(\mathcal{B})$ and its dual $\left(\mathcal{B}_{D}\right)$.

Vazirani [28] were the first to show a non-trivial integrality gap upper bound of $3 / 2$ on quasibipartite graphs; this was subsequently improved to $4 / 3$ by Chakrabarty et al. [4], who gave another alternate formulation for $(\mathcal{B})$.

\subsubsection{Hypergraphic Relaxations}

Given a Steiner tree $T$, a full component of $T$ is a maximal subtree of $T$ all of whose leaves are terminals and all of whose internal nodes are Steiner nodes. The edge set of any Steiner tree can be partitioned in a unique way into full components by splitting at internal terminals; see Figure 2 on page 4 for an example.
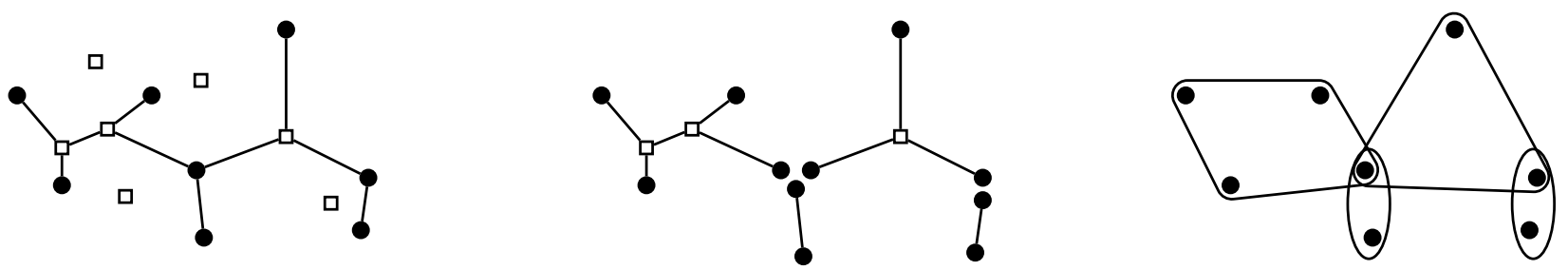

Figure 2: Black nodes are terminals and white nodes are Steiner nodes. Left: a Steiner tree for this instance. Middle: the Steiner tree's edges are partitioned into full components; there are four full components. Right: the hyperedges corresponding to these full components.

Let $\mathcal{K}$ be the set of all nonempty subsets of terminals (hyperedges). We associate with each $K \in \mathcal{K}$ a fixed full component spanning the terminals in $K$, and let $C_{K}$ be its cost $^{3}$. The problem of finding a minimum-cost Steiner tree spanning $R$ now reduces to that of finding a minimum-cost hyper-spanning tree in the hypergraph $(R, \mathcal{K})$.

Spanning trees in (normal) graphs are well understood and there are many different exact LP relaxations for this problem. These exact LP relaxations for spanning trees in graphs inspire the hypergraphic relaxations for the Steiner tree problem. Such relaxations have a variable $x_{K}$ for every ${ }^{4}$ $K \in \mathcal{K}$, and the different relaxations are based on the constraints used to capture a hyper-spanning tree, just as constraints on edges are used to capture a spanning tree in a graph.

The oldest hypergraphic LP relaxation is the subtour LP introduced by Warme [35] which is inspired by Edmonds' subtour elimination LP relaxation [12] for the spanning tree polytope. This LP relaxation uses the fact that there are no hypercycles in a hyper-spanning tree, and that it is spanning. More formally, let $\rho(X):=\max (0,|X|-1)$ be the rank of a set $X$ of vertices. Then a subhypergraph $\left(R, \mathcal{K}^{\prime}\right)$ is a hyper-spanning tree iff $\sum_{K \in \mathcal{K}^{\prime}} \rho(K)=\rho(R)$ and $\sum_{K \in \mathcal{K}^{\prime}} \rho(K \cap S) \leq \rho(S)$

\footnotetext{
${ }^{3}$ We choose the minimum cost full component if there are many. If there is no full component spanning $K$, we let $C_{K}$ be infinity. Such a minimum cost component can be found in polynomial time, if $|K|$ is a constant.

${ }^{4}$ Observe that there could be exponentially many hyperedges. This computational issue is circumvented by considering hyperedges of size at most $r$, for some constant $r$. By a result of Borchers and Du [2], this leads to only a $(1+\Theta(1 / \log r))$ factor increase in the optimal Steiner tree cost.
} 
for every subset $S$ of $R$. The corresponding LP relaxation, denoted below as $(\mathcal{S})$, is called the subtour elimination LP relaxation.

$$
\begin{aligned}
\min \left\{\sum_{K \in \mathcal{K}} C_{K} x_{K}:\right. & x \in \mathbf{R}_{\geq 0}^{\mathcal{K}}, \quad \sum_{K \in \mathcal{K}} x_{K} \rho(K)=\rho(R), \\
& \left.\sum_{K \in \mathcal{K}} x_{K} \rho(K \cap S) \leq \rho(S), \forall S \subset R\right\}
\end{aligned}
$$

Warme showed that if the maximum hyperedge size $r$ is bounded by a constant, the LP can be solved in polynomial time.

The next hypergraphic LP introduced for Steiner tree was a directed hypergraph formulation $(\mathcal{D})$, introduced by Polzin and Vahdati Daneshmand [26], and inspired by the bidirected cut relaxation. Given a full component $K$ and a terminal $i \in K$, let $K^{i}$ denote the arborescence obtained by directing all the edges of $K$ towards $i$. Think of this as directing the hyperedge $K$ towards $i$ to get the directed hyperedge $K^{i}$. Vertex $i$ is called the head of $K^{i}$ while the terminals in $K \backslash i$ are the tails of $K$. The cost of each directed hyperedge $K^{i}$ is the cost of the corresponding undirected hyperedge $K$. In the directed hypergraph formulation, there is a variable $x_{K^{i}}$ for every directed hyperedge $K^{i}$. As in the bidirected cut relaxation, there is a vertex $r \in R$ which is a root, and as described above, a subset $U \subseteq R$ of terminals is valid if it does not contain the root but contains at least one vertex in $R$. We let $\Delta^{\text {out }}(U)$ be the set of directed full components coming out of $U$, that is all $K^{i}$ such that $U \cap K \neq \varnothing$ but $i \notin U$. Let $\overrightarrow{\mathcal{K}}$ be the set of all directed hyperedges. We show the directed hypergraph relaxation and its dual in Figure 3.

$$
\begin{aligned}
& \min \left\{\sum_{K \in \mathcal{K}, i \in K} C_{K} x_{K^{i}}: x \in \mathbf{R}_{\geq 0}^{\overrightarrow{\mathcal{K}}}\right. \\
& \left.\sum_{K^{i} \in \Delta^{\text {out }}(U)} x_{K^{i}} \geq 1, \quad \forall \text { valid } U \subseteq R\right\}
\end{aligned}
$$

Figure 3: The directed hypergraph relaxation $(\mathcal{D})$ and its dual $\left(\mathcal{D}_{D}\right)$.

Polzin \& Vahdati Daneshmand [26] showed that $\mathrm{OPT}(\mathcal{D})=\mathrm{OPT}(\mathcal{S})$. Moreover they observed that this directed hypergraphic relaxation strengthens the bidirected cut relaxation.

Lemma 1.2 ([26]). For any instance, $\operatorname{OPT}(\mathcal{D}) \geq \operatorname{OPT}(\mathcal{B})$.

Proof sketch. It suffices to show that any solution $x$ of $(\mathcal{D})$ can be converted to a feasible solution $x^{\prime}$ of $(\mathcal{B})$ of the same cost. For each arc $a$, let $x_{a}^{\prime}$ be the sum of $x_{K^{i}}$ over all directed full components $K^{i}$ that (when viewed as an arborescence) contain $a$. Now for any valid subset $U$ of $V$, it is not hard to see that every directed full component leaving $R \cap U$ has at least one arc leaving $U$, hence $\sum_{a \in \delta^{\text {out }}(U)} x_{a}^{\prime} \geq \sum_{K^{i} \in \Delta^{\text {out }}(R \cap U)} x_{K^{i}} \geq 1$ and $x^{\prime}$ is feasible as needed.

See [26] for an example where the strict inequality $\operatorname{OPT}(\mathcal{D})>\operatorname{OPT}(\mathcal{B})$ holds.

Könemann et al. [23], inspired by the work of Chopra [6], described a partition-based relaxation which captures that given any partition of the terminals, any hyper-spanning tree must have sufficiently many "cross hyperedges". More formally, a partition, $\pi$, is a collection of pairwise disjoint nonempty terminal sets $\left(\pi_{1}, \ldots, \pi_{q}\right)$ whose union equals $R$. The number of parts $q$ of $\pi$ is referred to as the partition's rank and denoted as $r(\pi)$. Let $\Pi_{R}$ be the set of all partitions of $R$. Given a partition $\pi=\left\{\pi_{1}, \ldots, \pi_{q}\right\}$, define the rank contribution $\mathrm{rc}_{K}^{\pi}$ of hyperedge 
$K \in \mathcal{K}$ for $\pi$ as the rank reduction of $\pi$ obtained by merging the parts of $\pi$ that are touched by $K$; i.e., $\mathrm{rc}_{K}^{\pi}:=\left|\left\{i: K \cap \pi_{i} \neq \varnothing\right\}\right|-1$. Then a hyper-spanning tree $\left(R, \mathcal{K}^{\prime}\right)$ must satisfy $\sum_{K \in \mathcal{K}^{\prime}} \mathrm{rc}_{K}^{\pi} \geq r(\pi)-1$. The partition based LP of [23] and its dual are given in Figure 4 on page 6.

$$
\begin{aligned}
& \min \left\{\sum_{K \in \mathcal{K}} C_{K} x_{K}: \quad x \in \mathbf{R}_{\geq 0}^{\mathcal{K}}\right. \\
& \left.\sum_{K \in \mathcal{K}} x_{K} \mathrm{rc}_{K}^{\pi} \geq r(\pi)-1, \quad \forall \pi \in \Pi_{R}\right\} \\
& \begin{array}{r|r}
(\mathcal{P}) \quad \max \left\{\sum_{\pi}(r(\pi)-1) \cdot y_{\pi}: \quad y \in \mathbf{R}_{\geq 0}^{\Pi_{R}}\right. \\
\left.\sum_{\pi \in \Pi_{R}} y_{\pi} \mathrm{rc}_{K}^{\pi} \leq C_{K}, \quad \forall K \in \mathcal{K}\right\}
\end{array}
\end{aligned}
$$

Figure 4: The unbounded partition relaxation $(\mathcal{P})$ and its dual $\left(\mathcal{P}_{D}\right)$.

The feasible region of $(\mathcal{P})$ is unbounded, since if $x$ is a feasible solution for $(\mathcal{P})$ then so is any $x^{\prime} \geq x$. We obtain a bounded partition LP relaxation, denoted by $\left(\mathcal{P}^{\prime}\right)$ and shown below, by adding a valid equality constraint to the LP.

$$
\min \left\{\sum_{K \in \mathcal{K}} C_{K} x_{K}: x \in(\mathcal{P}), \sum_{K \in \mathcal{K}} x_{K}(|K|-1)=|R|-1\right\}
$$

\subsubsection{Discussion of Computational Issues}

The bidirected cut relaxation is very attractive from a perspective of computational implementation. Although the formulation given in Section 1.2.1 has an exponential number of constraints, an equivalent compact flow formulation with $O(|E||R|)$ variables and constraints is well-known.

What is known regarding solving the hypergraphic LPs? They are good enough to get theoretical results but less attractive in practice, as we now explain. Using a separation oracle, Warme showed [35] that for any chosen family $\mathcal{K}$ of full components, the subtour LP can be optimized in time poly $(|V|,|\mathcal{K}|)$. For the common $r$-restricted setting of $\mathcal{K}$ to be all possible full components of size at most $r$ for constant $r$, we have $\mathcal{K} \leq\left(\begin{array}{c}|R| \\ r\end{array}\right)$. This is polynomial for any fixed $r$, and the relative error caused by this choice of $r$ is at most the $r$-Steiner ratio $\rho_{r}=1+\Theta(1 / \log r)$ [2]. But this is not so practical: to get relative error $1+\epsilon$, we apply the ellipsoid algorithm to an LP with $|R|^{\exp (\Theta(1 / \epsilon))}$ variables!

In the unrestricted setting where $\mathcal{K}$ contains all possible full components without regard to size, it is an open problem to optimize any of the hypergraphic LPs exactly in polynomial time. We make some progress here: in quasibipartite instances, the proof method of our hypergraphic-bidirected equivalence theorem (Section 3.3) implies that one can exactly compute the LP optimal value, and a dual optimal solution. Regarding this open problem, we note that the $r$-restricted LP optimum is at most $\rho_{r}$ times the unrestricted optimum, and wonder whether there might be some advantage gained by using the fact that the hypergraphic LPs have sparse optima.

We reiterate our feeling that it is important to obtain practical algorithms and understand the bidirected cut relaxation as well as possible, e.g. we know now that it has an integrality gap of at most 1.28 on quasi-bipartite instances, but obtaining such a bound directly could give new insights.

\subsubsection{Other Related Work}

In the special case of $r$-restricted instances for $r=3$, the partition hypergraphic LP is essentially a special case of an LP introduced by Vande Vate [33] for matroid matching, which is totally dual 
half-integral [16]. Additional facts about the hypergraphic relaxations appear in the thesis of the third author [27], e.g. a combinatorial "gainless tree formulation" for the LPs similar in flavour to the "1-tree bound" for the Held-Karp TSP relaxation.

\section{Uncrossing Partitions}

In this section we are interested in uncrossing a minimal set of tight partitions that uniquely define a basic feasible solution to $(\mathcal{P})$. We start with a few preliminaries necessary to state our result formally.

\section{$2.1 \quad$ Preliminaries}

We introduce some needed well-known properties of partitions that arise in combinatorial lattice theory [32].

Definition 2.1. We say that a partition $\pi^{\prime}$ refines another partition $\pi$ if each part of $\pi^{\prime}$ is contained in some part of $\pi$. We also say $\pi$ coarsens $\pi^{\prime}$. Two partitions cross if neither refines the other. A family of partitions forms a chain if no pair of them cross. Equivalently, a chain is any family $\pi^{1}, \pi^{2}, \ldots, \pi^{t}$ such that $\pi^{i}$ refines $\pi^{i-1}$ for each $1<i \leq t$.

The family $\Pi_{R}$ of all partitions of $R$ forms a lattice with a meet operator $\wedge: \Pi_{R}^{2} \rightarrow \Pi_{R}$ and a join operator $\vee: \Pi_{R}^{2} \rightarrow \Pi_{R}$. The meet $\pi \wedge \pi^{\prime}$ is the coarsest partition that refines both $\pi$ and $\pi^{\prime}$, and the join $\pi \vee \pi^{\prime}$ is the most refined partition that coarsens both $\pi$ and $\pi^{\prime}$. See Figure 5 on page 7 for an illustration.

Definition 2.2 (Meet of partitions). Let the parts of $\pi$ be $\pi_{1}, \ldots, \pi_{t}$ and let the parts of $\pi^{\prime}$ be $\pi_{1}^{\prime}, \ldots, \pi_{u}^{\prime}$. Then the parts of the meet $\pi \wedge \pi^{\prime}$ are the nonempty intersections of parts of $\pi$ with parts of $\pi^{\prime}$,

$$
\pi \wedge \pi^{\prime}=\left\{\pi_{i} \cap \pi_{j}^{\prime} \mid 1 \leq i \leq t, 1 \leq j \leq u \text { and } \pi_{i} \cap \pi_{j}^{\prime} \neq \varnothing\right\} .
$$

Given a graph $G$ and a partition $\pi$ of $V(G)$, we say that $G$ induces $\pi$ if the parts of $\pi$ are the vertex sets of the connected components of $G$.

Definition 2.3 (Join of partitions). Let $(R, E)$ be a graph that induces $\pi$, and let $\left(R, E^{\prime}\right)$ be a graph that induces $\pi^{\prime}$. Then the graph $\left(R, E \cup E^{\prime}\right)$ induces $\pi \vee \pi^{\prime}$.

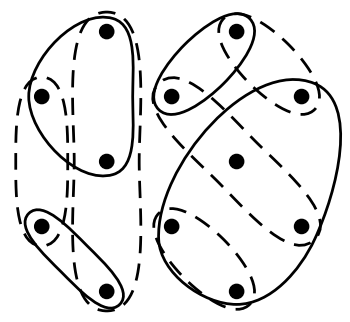

(a)

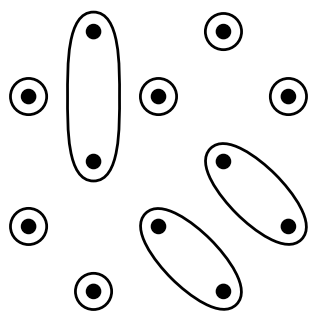

(b)

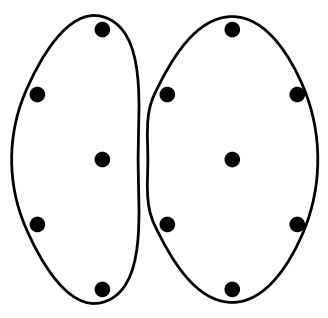

(c)

Figure 5: Illustrations of some partitions. The black dots are the terminal set $R$. (a): two partitions; neither refines the other. (b): the meet of the partitions from (a). (c): the join of the partitions from (a). 
Given a feasible solution $x$ to $(\mathcal{P})$, a partition $\pi$ is tight if $\sum_{K \in \mathcal{K}} x_{K} \operatorname{rc}_{K}^{\pi}=r(\pi)-1$. Let tight $(x)$ be the set of all tight partitions. We are interested in uncrossing this set of partitions. More precisely, we wish to find a cross-free set of partitions (chain) which uniquely defines $x$. One way would be to prove the following.

Property 2.4. If two crossing partitions $\pi$ and $\pi^{\prime}$ are in tight $(x)$, then so are $\pi \wedge \pi^{\prime}$ and $\pi \vee \pi^{\prime}$.

This type of property is already well-used [9, 13, 21, 31] for sets (with meets and joins replaced by unions and intersections respectively), and the standard approach is the following. The typical proof considers the constraints in $(\mathcal{P})$ corresponding to $\pi$ and $\pi^{\prime}$ and uses the "supermodularity" of the RHS and the "submodularity" of the coefficients in the LHS. In particular, if the following is true,

$$
\begin{aligned}
& \forall \pi, \pi^{\prime}: r\left(\pi \vee \pi^{\prime}\right)+r\left(\pi \wedge \pi^{\prime}\right) \geq r(\pi)+r\left(\pi^{\prime}\right) \\
& \forall K, \pi, \pi^{\prime}: \mathrm{rc}_{K}^{\pi}+\mathrm{rc}_{K}^{\pi^{\prime}} \geq \mathrm{rc}_{K}^{\pi \vee \pi^{\prime}}+\mathrm{rc}_{K}^{\pi \wedge \pi^{\prime}}
\end{aligned}
$$

then Property 2.4 can be proved easily by writing a string of inequalities. ${ }^{5}$

Inequality (7) is indeed true (see, for example, [32]), but unfortunately inequality (8) is not true in general, as the following example shows.

Example 2.5. Let $R=\{1,2,3,4\}, \pi=\{\{1,2\},\{3,4\}\}$ and $\pi^{\prime}=\{\{1,3\},\{2,4\}\}$. Let $K$ denote the full component $\{1,2,3,4\}$. Then $\mathrm{rc}_{K}^{\pi}+\mathrm{rc}_{K}^{\pi^{\prime}}=1+1<0+3=\mathrm{rc}_{K}^{\pi \vee \pi^{\prime}}+\mathrm{rc}_{K}^{\pi \wedge \pi^{\prime}}$.

Nevertheless, Property 2.4 is true; its correct proof is given in Section 2.2 and depends on a simple though subtle extension of the usual approach. The crux of the insight needed to fix the approach is not to consider pairs of constraints in $(\mathcal{P})$, but rather multi-sets which may contain more than two inequalities. Using this uncrossing result, we can prove the following theorem (details are given in Section 2.3). Here, we let $\underline{\pi}$ denote $\{R\}$, the unique partition with (minimal) rank 1; later we use $\bar{\pi}$ to denote $\{\{r\} \mid r \in R\}$, the unique partition with (maximal) rank $|R|$.

Theorem 1. Let $x^{*}$ be a basic feasible solution of $(\mathcal{P})$, and let $\mathcal{C}$ be an inclusion-wise maximal chain in tight $\left(x^{*}\right) \backslash \underline{\pi}$. Then $x^{*}$ is uniquely defined by

$$
\sum_{K \in \mathcal{K}} \mathrm{rc}_{K}^{\pi} x_{K}^{*}=r(\pi)-1 \quad \forall \pi \in \mathcal{C}
$$

Any chain of distinct partitions of $R$ that does not contain $\underline{\pi}$ has size at most $|R|-1$, and this is an upper bound on the rank of the system in (9). Elementary linear programming theory immediately yields the following corollary.

Corollary 2.6. Any basic solution $x^{*}$ of $(\mathcal{P})$ has at most $|R|-1$ non-zero coordinates.

\subsection{Partition Uncrossing Inequalities}

We start with the following definition.

Definition 2.7. Let $\pi \in \Pi_{R}$ be a partition and let $S \subset R$. Define the merged partition $m(\pi, S)$ to be the most refined partition that coarsens $\pi$ and contains all of $S$ in a single part. See Figure 6 on page 9 for an example. Informally, $m(\pi, S)$ is obtained by merging all parts of $\pi$ which intersect $S$.

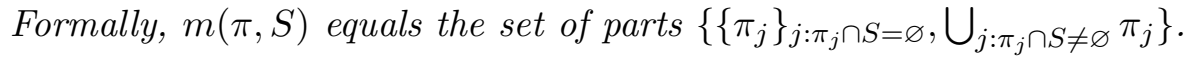



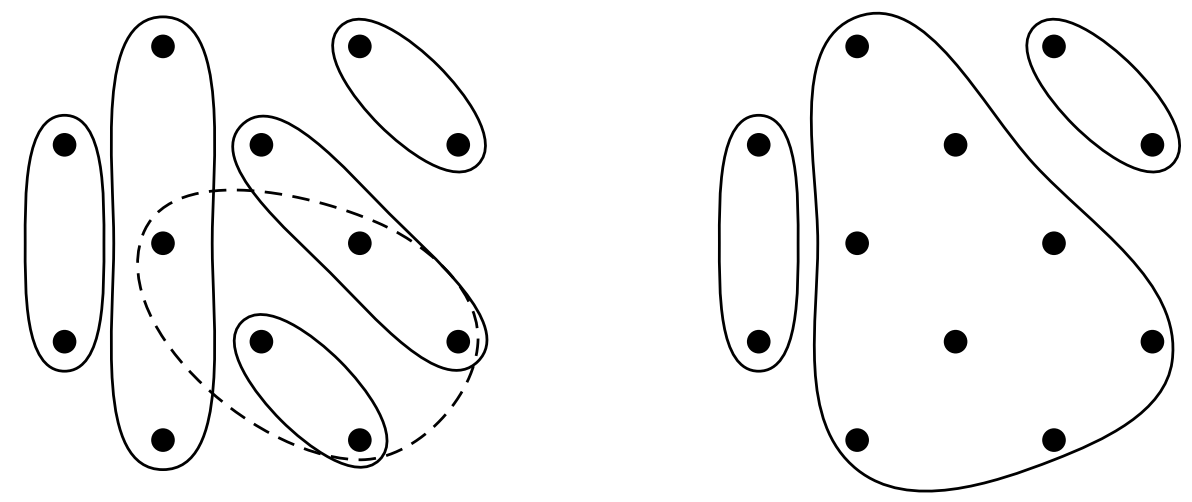

Figure 6: Illustration of merging. The left figure shows a (solid) partition $\pi$ along with a (dashed) set $S$. The right figure shows the merged partition $m(\pi, S)$.

We will use the following straightforward fact later:

$$
\mathrm{rc}_{K}^{\pi}=r(\pi)-r(m(\pi, K)) .
$$

We now state the (true) inequalities which replace the false inequality (8). Later, we show how one uses these to obtain partition uncrossing, e.g. to prove Property 2.4 .

Lemma 2.8 (Partition Uncrossing Inequalities). Let $\pi, \pi^{\prime} \in \Pi_{R}$ and let the parts of $\pi$ be $\pi_{1}, \pi_{2}, \ldots, \pi_{r(\pi)}$.

$$
\begin{aligned}
& r(\pi)\left[r\left(\pi^{\prime}\right)-1\right]+[r(\pi)-1]=\left[r\left(\pi \wedge \pi^{\prime}\right)-1\right]+\sum_{i=1}^{r(\pi)}\left[r\left(m\left(\pi^{\prime}, \pi_{i}\right)\right)-1\right] \\
& \forall K \in \mathcal{K}: \quad r(\pi)\left[\mathrm{rc}_{K}^{\pi^{\prime}}\right]+\left[\mathrm{rc}_{K}^{\pi}\right] \geq\left[\mathrm{rc}_{K}^{\pi \wedge \pi^{\prime}}\right]+\sum_{i=1}^{r(\pi)}\left[\mathrm{rc}_{K}^{m\left(\pi^{\prime}, \pi_{i}\right)}\right]
\end{aligned}
$$

Before giving the proof of the above lemma, let us first show how it can be used to prove the statement Property 2.4.

Proof of Property 2.4. Since $\pi$ and $\pi^{\prime}$ are tight,

$$
\begin{array}{r}
r(\pi)\left[r\left(\pi^{\prime}\right)-1\right]+[r(\pi)-1]=r(\pi)\left[\sum_{K} x_{K} \mathrm{rc}_{K}^{\pi^{\prime}}\right]+\left[\sum_{K} x_{K} \mathrm{rc} \mathrm{c}_{K}^{\pi}\right]=\sum_{K} x_{K}\left(r(\pi)\left[\mathrm{rc}_{K}^{\pi^{\prime}}\right]+\left[\mathrm{rc}_{K}^{\pi}\right]\right) \\
\geq \sum_{K} x_{K}\left(\left[\mathrm{rc}_{K}^{\pi \wedge \pi^{\prime}}\right]+\sum_{i=1}^{r(\pi)}\left[\mathrm{rc}_{K}^{m\left(\pi^{\prime}, \pi_{i}\right)}\right]\right)=\sum_{K} x_{K}\left[\mathrm{rc}_{K}^{\pi \wedge \pi^{\prime}}\right]+\sum_{i=1}^{r(\pi)} \sum_{K} x_{K}\left[\mathrm{rc}_{K}^{m\left(\pi^{\prime}, \pi_{i}\right)}\right] \\
\geq\left[r\left(\pi \wedge \pi^{\prime}\right)-1\right]+\sum_{i=1}^{r(\pi)}\left[r\left(m\left(\pi^{\prime}, \pi_{i}\right)\right)-1\right]=r(\pi)\left[r\left(\pi^{\prime}\right)-1\right]+[r(\pi)-1]
\end{array}
$$

where the first inequality follows from (12) and the second from (5) (as $x$ is feasible); the last equality is (11). Since the first and last terms are equal, all the inequalities are equalities, in particular

\footnotetext{
${ }^{5}$ In this hypothetical scenario we get $r(\pi)+r\left(\pi^{\prime}\right)-2=\sum_{K} x_{K}\left(\mathrm{rc}_{K}^{\pi}+\mathrm{rc}_{K}^{\pi^{\prime}}\right) \geq \sum_{K} x_{K}\left(\mathrm{rc}_{K}^{\pi \wedge \pi^{\prime}}+\mathrm{rc}_{K}^{\pi \vee \pi^{\prime}}\right) \geq$ $r\left(\pi \wedge \pi^{\prime}\right)+r\left(\pi \vee \pi^{\prime}\right)-2 \geq r(\pi)+r\left(\pi^{\prime}\right)-2$; thus the inequalities hold with equality, and the middle one shows $\pi \wedge \pi^{\prime}$ and $\pi \vee \pi^{\prime}$ are tight.
} 
our application of (5) shows that $\pi \wedge \pi^{\prime}$ and each $m\left(\pi^{\prime}, \pi_{i}\right)$ is tight. Iterating the latter fact, we see that $m\left(\cdots m\left(m\left(\pi^{\prime}, \pi_{1}\right), \pi_{2}\right), \cdots\right)=\pi \vee \pi^{\prime}$ is also tight.

To prove the inequalities in Lemma 2.8 we need the following lemma that relates the rank of sets and the rank contribution of partitions. Recall $\rho(X):=\max (0,|X|-1)$.

Lemma 2.9. For a partition $\pi=\left\{\pi_{1}, \ldots, \pi_{t}\right\}$ of $R$, where $t=r(\pi)$, and for any $K \subseteq R$, we have

$$
\rho(K)=\mathrm{rc}_{K}^{\pi}+\sum_{i=1}^{t} \rho\left(K \cap \pi_{i}\right) .
$$

Proof. By definition, $K \cap \pi_{i} \neq \varnothing$ for exactly $1+\mathrm{rc}_{K}^{\pi}$ values of $i$. Also, $\rho\left(K \cap \pi_{i}\right)=0$ for all other $i$. Hence

$$
\sum_{i=1}^{t} \rho\left(K \cap \pi_{i}\right)=\sum_{i: K \cap \pi_{i} \neq \varnothing}\left(\left|K \cap \pi_{i}\right|-1\right)=\left(\sum_{i: K \cap \pi_{i} \neq \varnothing}\left|K \cap \pi_{i}\right|\right)-\left(\mathrm{rc}_{K}^{\pi}+1\right) .
$$

Observe that $\sum_{i: K \cap \pi_{i} \neq \varnothing}\left|K \cap \pi_{i}\right|=|K|=\rho(K)+1$; using this fact together with Equation (13) we obtain

$$
\sum_{i=1}^{t} \rho\left(K \cap \pi_{i}\right)=\left(\sum_{i: K \cap \pi_{i} \neq \varnothing}\left|K \cap \pi_{i}\right|\right)-\left(\mathrm{rc}_{K}^{\pi}+1\right)=\rho(K)-1+\left(\mathrm{rc}_{K}^{\pi}+1\right) .
$$

Rearranging, the proof of Lemma 2.9 is complete.

Proof of Lemma 2.8. First, we argue that $\pi \wedge \pi^{\prime}=\bar{\pi}$ holds without loss of generality. In the general case, for each part $p$ of $\pi \wedge \pi^{\prime}$ with $|p| \geq 2$, contract $p$ into one pseudo-vertex and define the new $K$ to include the pseudo-vertex corresponding to $p$ if and only if $K \cap p \neq \varnothing$. This contraction does not affect the value of any of the terms in Equations (12) and (11), so is without loss of generality. After contraction, for any part $\pi_{i}$ of $\pi$ and part $\pi_{j}^{\prime}$ of $\pi^{\prime}$, we have $\left|\pi_{i} \cap \pi_{j}^{\prime}\right| \leq 1$, so indeed $\pi \wedge \pi^{\prime}=\bar{\pi}$.

Proof of Equation (11). Fix $i$. Since $\left|\pi_{i} \cap \pi_{j}^{\prime}\right| \leq 1$ for all $j$, the rank contribution $\mathrm{rc}_{\pi_{i}} \pi^{\prime}$ is equal to $\left|\pi_{i}\right|-1$. Then using Equation (10) we know that $r\left(m\left(\pi^{\prime}, \pi_{i}\right)\right)=r\left(\pi^{\prime}\right)-\left|\pi_{i}\right|+1$. Thus adding over all $i$, the right-hand side of Equation (11) is equal to

$$
|R|-1+\sum_{i=1}^{r(\pi)}\left(r\left(\pi^{\prime}\right)-\left|\pi_{i}\right|\right)=|R|-1+r(\pi) r\left(\pi^{\prime}\right)-|R|
$$

and this is precisely the left-hand side of Equation (11).

Proof of Equation (12). Fix $i$. Since $\left|\pi_{i} \cap \pi_{j}^{\prime}\right| \leq 1$ for all $j$, we have

$$
\mathrm{rc}_{K}^{\pi^{\prime}}-\mathrm{rc}_{K}^{m\left(\pi^{\prime}, \pi_{i}\right)} \geq \rho\left(\pi_{i} \cap K\right)
$$

because, when we merge the parts of $\pi^{\prime}$ intersecting $\pi_{i}$, we make $K$ span at least $\rho\left(\pi_{i} \cap K\right)$ fewer parts. Note that the inequality could be strict if both $\pi_{i}$ and $K$ intersect a part of $\pi^{\prime}$ without having a common vertex in that part.

Adding the right-hand side of Equation (14) over all $i$ gives

$$
\sum_{i=1}^{r(\pi)}\left(\mathrm{rc}_{K}^{\pi^{\prime}}-\mathrm{rc}_{K}^{m\left(\pi^{\prime}, \pi_{i}\right)}\right) \geq \sum_{i=1}^{r(\pi)} \rho\left(\pi_{i} \cap K\right)=\rho(K)-\mathrm{rc}_{K}^{\pi}
$$


where the last equality follows from Lemma 2.9. To finish the proof we observe $\rho(K)=\mathrm{rc}_{K}^{\pi \wedge \pi^{\prime}}$, since $\pi \wedge \pi^{\prime}=\bar{\pi}$.

This completes the proof of Lemma 2.8.

\subsection{Sparsity of Basic Feasible Solutions: Proof of Theorem 1}

Proof. Let $\operatorname{supp}\left(x^{*}\right)$ be the full components $K$ with $x_{K}^{*}>0$. Consider the constraint submatrix with rows corresponding to the tight partitions and columns corresponding to the full components in $\operatorname{supp}\left(x^{*}\right)$. Since $x^{*}$ is a basic feasible solution, any full-rank subset of rows uniquely defines $x^{*}$. We now show that any maximal chain $\mathcal{C}$ in tight $\left(x^{*}\right)$ corresponds to such a subset.

Let $\operatorname{row}(\pi) \in \mathbf{R}^{\operatorname{supp}\left(x^{*}\right)}$ denote the row corresponding to partition $\pi$ of this matrix, i.e., $\operatorname{row}(\pi)_{K}=$ $\mathrm{rc}_{K}^{\pi}$, and given a collection $\mathcal{R}$ of partitions (rows), let $\operatorname{span}(\mathcal{R})$ denote the linear span of the rows in $\mathcal{R}$. We now prove that for any tight partition $\pi \notin \mathcal{C}$, we have $\operatorname{row}(\pi) \in \operatorname{span}(\mathcal{C})$; this will complete the proof of the theorem.

For sake of contradiction, suppose $\operatorname{row}(\pi) \notin \operatorname{span}(\mathcal{C})$. Choose $\pi$ to be the counterexample partition with smallest rank $r(\pi)$. Firstly, since $\mathcal{C}$ is maximal, $\pi$ must cross some partition $\sigma$ in $\mathcal{C}$. Choose $\sigma$ to be the most refined partition in $\mathcal{C}$ which crosses $\pi$. Let the parts of $\sigma$ be $\left(\sigma_{1}, \ldots, \sigma_{t}\right)$. The following claim uses the partition uncrossing inequalities to derive a linear dependence between the rows corresponding to $\sigma, \pi$ and the partitions formed by merging parts of $\sigma$ with $\pi$.

Claim 2.10. We have $\operatorname{row}(\sigma)+|r(\sigma)| \cdot \operatorname{row}(\pi)=\operatorname{row}(\pi \wedge \sigma)+\sum_{i=1}^{t} \operatorname{row}\left(m\left(\pi, \sigma_{i}\right)\right)$.

Proof. Since $\sigma$ and $\pi$ are both tight partitions, the proof of Property 2.4 shows that the partition inequality (12) holds with equality for all $K \in \operatorname{supp}\left(x^{*}\right), \pi$ and $\sigma$, implying the claim.

Let $\mathrm{cp}_{\pi}(\sigma)$ be the parts of $\sigma$ which intersect at least two parts of $\pi$; i.e., merging the parts of $\pi$ that intersect $\sigma_{i}$, for any $\sigma_{i} \in \mathrm{cp}_{\pi}(\sigma)$, decreases the rank of $\pi$. Formally,

$$
\mathrm{cp}_{\pi}(\sigma):=\left\{\sigma_{i} \in \sigma: m\left(\pi, \sigma_{i}\right) \neq \pi\right\}
$$

Note that one can modify Claim 2.10 by subtracting $\left(r(\sigma)-\left|\mathrm{cp}_{\pi}(\sigma)\right|\right)$ row $(\pi)$ from both sides to get

$$
\operatorname{row}(\sigma)+\left|\operatorname{cp}_{\pi}(\sigma)\right| \cdot \operatorname{row}(\pi)=\operatorname{row}(\pi \wedge \sigma)+\sum_{\sigma_{i} \in \mathrm{cp}_{\pi}(\sigma)} \operatorname{row}\left(m\left(\pi, \sigma_{i}\right)\right)
$$

Now if $\operatorname{row}(\pi) \notin \operatorname{span}(\mathcal{C})$, we must have either $\operatorname{row}(\pi \wedge \sigma)$ is not in $\operatorname{span}(\mathcal{C})$ or $\operatorname{row}\left(m\left(\pi, \sigma_{i}\right)\right)$ is not in $\operatorname{span}(\mathcal{C})$ for some $i$. We show that either case leads to the needed contradiction, which will prove the theorem.

Case 1: $\operatorname{row}(\pi \wedge \sigma) \notin \operatorname{span}(\mathcal{C})$. Note there is $\sigma^{\prime} \in \mathcal{C}$ which crosses $\pi \wedge \sigma$, since $\pi \wedge \sigma$ is not in the maximal chain $\mathcal{C}$. Since $\sigma^{\prime}, \sigma \in \mathcal{C}$ and by considering the refinement order, it is easy to see that $\sigma^{\prime}$ (strictly) refines $\sigma$ and $\sigma^{\prime}$ crosses $\pi$. This contradicts our choice of $\sigma$ as the most refined partition in $\mathcal{C}$ crossing $\pi$, since $\sigma^{\prime}$ was also a candidate.

Case 2: $\operatorname{row}\left(m\left(\pi, \sigma_{i}\right)\right) \notin \operatorname{span}(\mathcal{C})$. Note $m\left(\pi, \sigma_{i}\right)$ is also tight. Since $\sigma_{i} \in \mathrm{cp}_{\pi}(\sigma), m\left(\pi, \sigma_{i}\right)$ has smaller rank than $\pi$. This contradicts our choice of $\pi$.

This completes the proof of Theorem 1. 


\section{Equivalence of Formulations}

In this section we describe our equivalence results. A summary of the known and new results is given in Figure 7 on page 12.

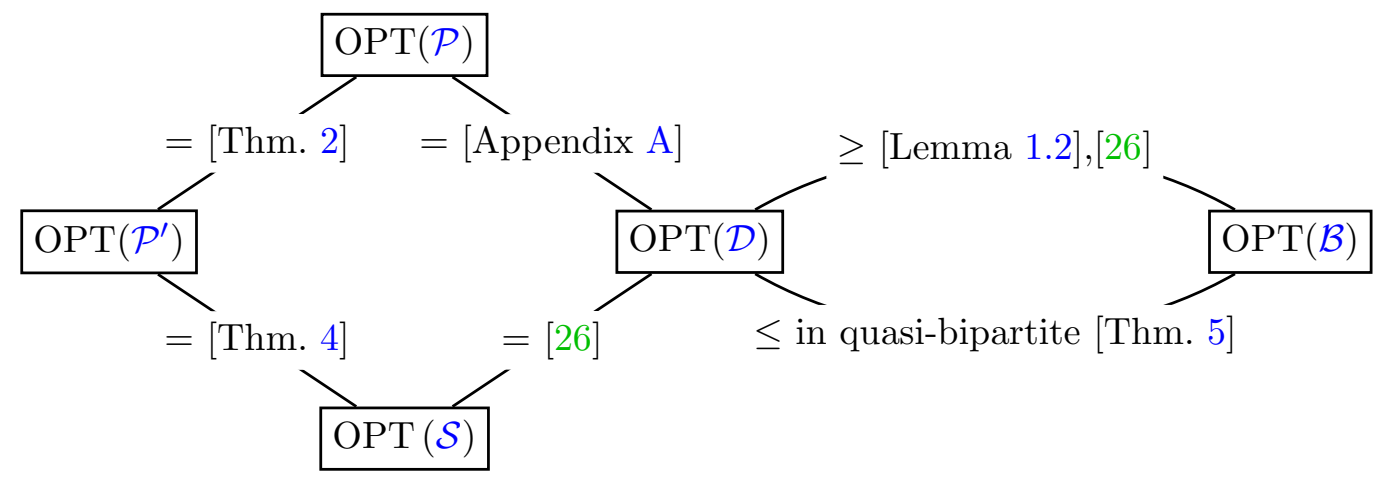

Figure 7: Summary of relations among various LP relaxations

As we mentioned in the introduction, we give a redundant set of proofs for completeness and to demonstrate novel techniques. The proof that $(\mathcal{P})$ and $(\mathcal{D})$ have the same value, which appears in Appendix A, is a consequence of hypergraph orientation results of Frank et al. [14].

\subsection{Bounded and Unbounded Partition Relaxations}

Theorem 2. The LPs $\left(\mathcal{P}^{\prime}\right)$ and $(\mathcal{P})$ have the same optimal value.

We actually prove a stronger statement.

Definition 3.1. The collection $\mathcal{K}$ of hyperedges is down-closed if whenever $S \in \mathcal{K}$ and $\varnothing \neq T \subset S$, then $T \in \mathcal{K}$. For down-closed $\mathcal{K}$, the cost function $C: \mathcal{K} \rightarrow \mathbf{R}_{+}$is non-decreasing if $C_{S} \leq C_{T}$ whenever $S \subset T$.

Theorem 3. If the set of hyperedges is down-closed and the cost function is non-decreasing, then $\left(\mathcal{P}^{\prime}\right)$ and $(\mathcal{P})$ have the same optimal value.

Theorem 3 implies Theorem 2 since the hypergraph and cost function derived from instances of the Steiner tree problem are down-closed and non-decreasing (e.g. $C_{\{k\}}=0$ for every $k \in R$; we remark that the variables $x_{\{k\}}$ act just as placeholders). Our proof of Theorem 2 relies on the following operation which we call shrinking.

Definition 3.2. Given an assignment $x: \mathcal{K} \rightarrow \mathbf{R}_{+}$to the full components, suppose $x_{K}>0$ for some $K$. The operation $\operatorname{Shrink}\left(x, K, K^{\prime}, \delta\right)$, where $K^{\prime} \subseteq K,\left|K^{\prime}\right|=|K|-1$ and $0<\delta \leq x_{K}$, changes $x$ to $x^{\prime}$ by decreasing $x_{K}^{\prime}:=x_{K}-\delta$ and increasing $x_{K^{\prime}}^{\prime}:=x_{K^{\prime}}+\delta$.

Note that shrinking is defined only for down-closed hypergraphs. Also note that on performing a shrinking operation, the cost of the solution cannot increase, if the cost function is nondecreasing. The theorem is proved by taking the optimum solution to $(\mathcal{P})$ which minimizes the sum $\sum_{K \in \mathcal{K}} x_{K}|K|$, and then showing that this must satisfy the equality in $\left(\mathcal{P}^{\prime}\right)$, or a shrinking operation can be performed. Now we give the details.

Proof of Theorem 3. It suffices to exhibit an optimum solution of $(\mathcal{P})$ which satisfies the equality in $\left(\mathcal{P}^{\prime}\right)$. Let $x$ be an optimal solution to $(\mathcal{P})$ which minimizes the sum $\sum_{K \in \mathcal{K}} x_{K}|K|$. 
Claim 3.3. For every $K$ with $x_{K}>0$ and for every $r \in K$, there exists a tight partition (w.r.t. $x$ ) $\pi$ such that the part of $\pi$ containing $r$ contains no other vertex of $K$.

Proof. Let $K^{\prime}=K \backslash\{r\}$. If the above is not true, then this implies that for every tight partition $\pi$, we have $\mathrm{rc}_{K}^{\pi}=\mathrm{rc}_{K^{\prime}}^{\pi}$. We now claim that there is a $\delta>0$ such that we can perform $\operatorname{Shrink}\left(x, K, K^{\prime}, \delta\right)$ while retaining feasibility in $(\mathcal{P})$. This is a contradiction since the shrink operation strictly reduces $\sum_{K}|K| x_{K}$ and doesn't increase cost. Specifically, take

$$
\delta:=\min \left\{x_{K}, \min _{\pi: \mathrm{rc}_{K^{\prime}}^{\pi}} \neq \mathrm{rc}_{K}^{\pi} \sum_{K} \mathrm{rc}_{K}^{\pi} x_{K}-r(\pi)+1\right\}
$$

which is positive since for tight partitions we have $\mathrm{rc}_{K}^{\pi}=\mathrm{rc}_{K^{\prime}}^{\pi}$.

Let $\operatorname{tight}(x)$ be the set of tight partitions, and $\pi^{*}:=\bigwedge\{\pi \mid \pi \in \operatorname{tight}(x)\}$ the meet of all tight partitions. By Property 2.4, $\pi^{*}$ is tight. By Claim 3.3, for any $K$ with $x_{K}>0$, we have $\mathrm{rc}_{K}^{\pi^{*}}=|K|-1$. Thus, $r\left(\pi^{*}\right)-1=\sum_{K \in \mathcal{K}} x_{K} \mathrm{rc}_{K}^{\pi^{*}}=\sum_{K \in \mathcal{K}} x_{K}(|K|-1) \geq r(\bar{\pi})-1$. But since $\bar{\pi}$ is the unique maximal-rank partition, this implies $\pi^{*}=\bar{\pi}$. Thus $\bar{\pi}$ is tight. This implies $x \in\left(\mathcal{P}^{\prime}\right)$.

\subsection{Partition and Subtour Elimination Relaxations}

Theorem 4. The feasible regions of $\left(\mathcal{P}^{\prime}\right)$ and $(\mathcal{S})$ are the same.

Proof. Let $x$ be any feasible solution to the $\operatorname{LP}(\mathcal{S})$. Note that the equality constraint of $\left(\mathcal{P}^{\prime}\right)$ is the same as that of $(\mathcal{S})$. We now show that $x$ satisfies $(5)$. Fix a partition $\pi=\left\{\pi_{1}, \ldots, \pi_{t}\right\}$, so $t=r(\pi)$. For each $1 \leq i \leq t$, subtract the inequality constraint in $(\mathcal{S})$ with $S=\pi_{i}$, from the equality constraint in $(\mathcal{S})$ to obtain

$$
\sum_{K \in \mathcal{K}} x_{K}\left(\rho(K)-\sum_{i=1}^{t} \rho\left(K \cap \pi_{i}\right)\right) \geq \rho(R)-\sum_{i=1}^{t} \rho\left(\pi_{i}\right) .
$$

From Lemma 2.9, $\rho(K)-\sum_{i=1}^{t} \rho\left(K \cap \pi_{i}\right)=\mathrm{rc}_{K}^{\pi}$. We also have $\rho(R)-\sum_{i=1}^{t} \rho\left(\pi_{i}\right)=|R|-1-$ $(|R|-r(\pi))=r(\pi)-1$. Thus $x$ is a feasible solution to the $\operatorname{LP}\left(\mathcal{P}^{\prime}\right)$.

Now, let $x$ be a feasible solution to $\left(\mathcal{P}^{\prime}\right)$ and it suffices to show that it satisfies the inequality constraints of $(\mathcal{S})$. Fix a set $S \subset R$. Note when $S=\varnothing$ that inequality constraint is vacuously true so we may assume $S \neq \varnothing$. Let $R \backslash S=\left\{r_{1}, \ldots, r_{u}\right\}$. Consider the partition $\pi=\left\{\left\{r_{1}\right\}, \ldots,\left\{r_{u}\right\}, S\right\}$. Subtract (5) for this $\pi$ from the equality constraint in $\left(\mathcal{P}^{\prime}\right)$, to obtain

$$
\sum_{K \in \mathcal{K}} x_{K}\left(\rho(K)-\mathrm{rc}_{K}^{\pi}\right) \leq \rho(R)-r(\pi)+1
$$

Using Lemma 2.9 and the fact that $\rho\left(K \cap\left\{r_{j}\right\}\right)=0$ (the set is either empty or a singleton), we get $\rho(K)-\mathrm{rc}_{K}^{\pi}=\rho(K \cap S)$. Finally, as $\rho(R)-r(\pi)+1=|R|-1-(|R \backslash S|+1)+1=\rho(S)$, the inequality $(18)$ is the same as the constraint needed. Thus $x$ is a feasible solution to $(\mathcal{S})$, proving the theorem.

\subsection{Partition and Bidirected Cut Relaxations in Quasibipartite Instances}

Theorem 5. On quasibipartite Steiner tree instances, $\operatorname{OPT}(\mathcal{B}) \geq \operatorname{OPT}(\mathcal{D})$.

To prove Theorem 5 , we look at the duals of the two LPs and we show $\operatorname{OPT}\left(\mathcal{B}_{D}\right) \geq \operatorname{OPT}\left(\mathcal{D}_{D}\right)$ in quasibipartite instances. Recall that the support of a solution to $\left(\mathcal{D}_{D}\right)$ is the family of sets with positive $z_{U}$. A family of sets is called laminar if for any two of its sets $A, B$ we have $A \subseteq B, B \subseteq A$, or $A \cap B=\varnothing$. 
Lemma 3.4. There exists an optimal solution to $\left(\mathcal{D}_{D}\right)$ whose support is a laminar family of sets.

Proof. Choose an optimal solution $z$ to $\left(\mathcal{D}_{D}\right)$ which maximizes $\sum_{U} z_{U}|U|^{2}$ among all optimal solutions. We claim that the support of this solution is laminar. Suppose not and there exists $U$ and $U^{\prime}$ with $U \cap U^{\prime} \neq \varnothing$ and $z_{U}>0$ and $z_{U^{\prime}}>0$. Define $z^{\prime}$ to be the same as $z$ except $z_{U}^{\prime}=z_{U}-\delta$, $z_{U^{\prime}}^{\prime}=z_{U^{\prime}}-\delta, z_{U \cup U^{\prime}}^{\prime}=z_{U \cup U^{\prime}}+\delta$ and $z_{U \cap U^{\prime}}^{\prime}=z_{U \cap U^{\prime}}+\delta$; we will show for small $\delta>0, z^{\prime}$ is feasible. Note that $U \cap U^{\prime}$ is not empty and $U \cup U^{\prime}$ doesn't contain $r$, and the objective value remains unchanged. Also note that for any $K$ and $i \in K$, if $z_{U \cup U^{\prime}}$ or $z_{U \cap U^{\prime}}$ appears in the summand of a constraint, then at least one of $z_{U}$ or $z_{U^{\prime}}$ also appears. If both $z_{U \cup U^{\prime}}$ and $z_{U \cap U^{\prime}}$ appears, then both $z_{U}$ and $z_{U^{\prime}}$ appears. Thus $z^{\prime}$ is an optimal solution and $\sum_{U} z_{U}^{\prime}|U|^{2}>\sum_{U} z_{U}|U|^{2}$, contradicting the choice of $z$.

Lemma 3.5. For quasibipartite instances, given a solution of $\left(\mathcal{D}_{D}\right)$ with laminar support, we can get a feasible solution to $\left(\mathcal{B}_{D}\right)$ of the same value.

Proof. This lemma is the heart of the theorem, and is a little technical to prove. We first give a sketch of how we convert a feasible solution $z$ of $\left(\mathcal{D}_{D}\right)$ into a feasible solution to $\left(\mathcal{B}_{D}\right)$ of the same value.

Comparing $\left(\mathcal{D}_{D}\right)$ and $\left(\mathcal{B}_{D}\right)$ one first notes that the former has a variable for every valid subset of the terminals, while the latter assigns values to all valid subsets of the entire vertex set. We say that an edge $u v$ is satisfied for a candidate solution $z$, if both a) $\sum_{U: u \in U, v \notin U} z_{U} \leq c_{u v}$ and b) $\sum_{U: v \in U, u \notin U} z_{U} \leq c_{u v}$ hold; $z$ is then feasible for $\left(\mathcal{B}_{D}\right)$ if all edges are satisfied.

Let $z$ be a feasible solution to $\left(\mathcal{D}_{D}\right)$. One easily verifies that all terminal-terminal edges are satisfied. On the other hand, terminal-Steiner edges may initially not be satisfied. To see this consider the Steiner vertex $v$ and its neighbours depicted in Figure 8 on page 14 below. Initially, none of the sets in $z$ 's support contains $v$, and the load on the edges incident to $v$ is quite skewed: the left-hand side of condition a) above may be large, while the left-hand side of condition b) is initially 0 .

To construct a valid solution for $\left(\mathcal{B}_{D}\right)$, we therefore lift the initial value $z_{S}$ of each terminal subset $S$ to supersets of $S$, by adding Steiner vertices. The lifting procedure processes each Steiner vertex $v$ one at a time; when processing $v$, we change $z$ by moving dual from some sets $U$ to $U \cup\{v\}$. Such a dual transfer decreases the left-hand side of condition a) for edge $u v$, and increases the (initially 0 ) left-hand sides of condition b) for edges connecting $v$ to neighbours other than $v$.

We will soon see that there is a way of carefully lifting duals around $v$ that ensures that all edges incident to $v$ become satisfied. The definition of our procedure will ensure that these edges remain satisfied for the rest of the lifting procedure. Since there are no Steiner-Steiner edges, all edges will be satisfied once all Steiner vertices are processed.

Throughout the lifting procedure, we will maintain that $z$ remains unchanged, when projected to the terminals. Formally, we maintain the following crucial projection invariant:

The quantity $\sum_{U: S \subseteq U \subseteq S \cup(V \backslash R)} z_{U}$

remains constant, for all terminal sets $S$.

This invariant leads to two observations: first, the constraint (4) is satisfied by $z$ at all times, even when it is defined on subsets of all vertices; second, $\sum_{U \subseteq V} z_{U}$ is constant throughout, and the objective value of $z$ in $\left(\mathcal{B}_{D}\right)$ is not affected by the lifting. The existence of a lifting of duals around Steiner vertex $v$ such

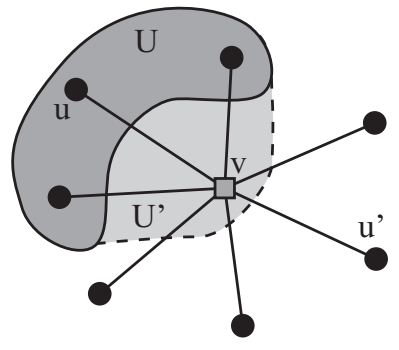

Figure 8: Lifting variable $z_{U}$. 
that (PI) is maintained, and such that all edges incident to $v$ are satisfied can be phrased as a feasibility problem for a linear system of inequalities. We will use Farkas' lemma and the feasibility of $z$ for (4) to complete the proof.

We now fill in the proof details. Let $\Gamma(v)$ denote the set of neighbours of vertex $v$ in the given graph $G$. In each iteration, where we process Steiner node $v$, let

$$
\mathcal{U}_{v}:=\left\{U: z_{U}>0 \text { and } U \cap \Gamma(v) \neq \varnothing\right\}
$$

be the sets in $z$ 's support that contain neighbours of $v$. Note that $U \in \mathcal{U}_{v}$ could contain Steiner vertices on which the lifting procedure has already taken place. However, by (PI) and by Lemma 3.4 the multi-family $\left\{U \cap R: U \in \mathcal{U}_{v}\right\}$ is laminar. In the lifting process, we will transfer $x_{U}$ units of the $z_{U}$ units of dual of each set $U \in \mathcal{U}_{v}$ to the set $U^{\prime}=U \cup\{v\}$; this decreases the dual load (LHS of (2)) on arcs from $U \cap \Gamma(v)$ to $v$ (e.g. $u v$ in Figure 8 on page 14) and increases the dual load on arcs from $v$ to $\Gamma(v) \backslash U$ (e.g. $v u^{\prime}$ in the figure). The following system of inequalities describes the set of feasible liftings.

$$
\begin{aligned}
\forall U \in \mathcal{U}_{v}: & x_{U} \leq z_{U} \\
\forall u \in \Gamma(v): & \sum_{U: u \in U}\left(z_{U}-x_{U}\right) \leq c_{u v} \\
\forall u \in \Gamma(v): & \sum_{U: u \notin U} x_{U} \leq c_{u v}
\end{aligned}
$$

Claim 3.6. If (L1), (L2), (L3) have a feasible solution $x \geq 0$, then the lifting procedure can be performed at Steiner vertex $v$, while maintaining the projection invariant property.

Proof. Define the new solution to be $z_{U}:=z_{U}-x_{U}$, and, $z_{(U \cup v)}:=x_{U}$, for all $U \in \mathcal{U}_{v}$, and $z_{U}$ remains unchanged for all other $U$. It is easy to check that all edges which were satisfied remain satisfied, and (L2) and (L3) imply that all edges incident to $v$ are satisfied. Also note that the projection invariant property is maintained.

By Farkas' lemma, if (L1), (L2), (L3) do not have a feasible solution $x \geq 0$, then there exist non-negative multipliers - $\lambda_{U}$ for all $U \in \mathcal{U}_{v}$, and $\alpha_{u}, \beta_{u}$ for all $u \in \Gamma(v)$ - satisfying the following dual set of linear inequalities:

$$
\begin{array}{r}
\sum_{U \in \mathcal{U}_{v}} \lambda_{U} z_{U}+\sum_{u \in \Gamma(v)} \alpha_{u}\left(c_{u v}-\sum_{U: u \in U} z_{U}\right)+\sum_{u \in \Gamma(v)} \beta_{u} c_{u v}<0 \\
\forall U \in \mathcal{U}_{v}: \lambda_{U}-\sum_{u \in U} \alpha_{u}+\sum_{u \notin U} \beta_{u} \geq 0
\end{array}
$$

As a technicality, note that the sub-system $\{(\mathrm{L} 1),(\mathrm{L} 2), x \geq 0\}$ is feasible - take $x=z$. Thus any $\alpha, \beta, \lambda$ satisfying (D1) and (D2) has $\sum_{u} \beta_{u}>0$, so by dividing all $\alpha, \beta, \lambda$ by $\sum_{i} \beta_{i}$, we may assume without loss of generality that

$$
\sum_{u \in \Gamma(v)} \beta_{u}=1
$$

Subtracting (D3) from (D2) allows us to rewrite the latter set of constraints conveniently as

$$
\forall U \in \mathcal{U}_{v}: \quad \lambda_{U}-\sum_{u \in U}\left(\alpha_{u}+\beta_{u}\right)+1 \geq 0 .
$$


The following claim shows that (L1), (L2), (L3) does have a feasible solution, and thus by Claim 3.6, lifting can be done, which completes the proof of Lemma 3.5.

Claim 3.7. There exists no feasible solution to $\{\alpha, \beta, \lambda \geq 0:(\mathrm{D} 1),(\mathrm{D} 2$ '), and (D3) $\}$.

Proof. Consider the linear program which minimizes the LHS of (D1) subject to the constraints (D2') and (D3). We show that the LP has value at least 0 , which will complete the proof.

Let $\left(\lambda^{*}, \alpha^{*}, \beta^{*}\right)$ be an optimal solution to the LP. In Lemma 3.8 we will show that the constraint matrix of the LP is totally unimodular; hence, since the right-hand side of the given system is integral, we may assume that $\lambda^{*}, \alpha^{*}$, and $\beta^{*}$ are non-negative and integral. From (D3) we infer

There is a unique $\bar{u} \in \Gamma(v)$ for which $\beta_{\bar{u}}^{*}=1$; for all $u \neq \bar{u}, \beta_{u}^{*}=0$.

Moreover, since each $\lambda_{U}$ appears only in the two constraints (D2') and $\lambda_{U} \geq 0$, and since $\lambda_{U}$ has nonnegative coefficient in the objective, we may assume

$$
\lambda_{U}^{*}=\lambda_{U}^{*}\left(\alpha^{*}, \beta^{*}\right):=\max \left\{\sum_{u \in U}\left(\alpha_{u}^{*}+\beta_{u}^{*}\right)-1,0\right\}
$$

for all $U$.

Next, we establish the following:

$$
\alpha_{u}^{*}+\beta_{u}^{*} \in\{0,1\} \text { for all } u \in \Gamma(v) .
$$

Suppose for the sake of contradiction that property (21) does not hold for our solution. Let $u$ be such that $\alpha_{u}^{*}+\beta_{u}^{*} \geq 2$. By (19), $\alpha_{u}^{*} \geq 1$. We propose the following update to our solution: decrease $\alpha_{u}^{*}$ by 1 (which by (20) will decrease $\lambda_{U}^{*}$ by 1 for all $U \in \mathcal{U}_{v}$ ). This maintains the feasibility of (D2'), and the objective value decreases by

$$
\sum_{U \in \mathcal{U}_{v}: u \in U} z_{U}+\left(c_{u v}-\sum_{u \in U} z_{U}\right)
$$

which is non-negative as $c \geq 0$. By repeating this operation, we may clearly ensure property (21).

Let $K \subseteq \Gamma(v)$ be the set $\left\{u \mid \alpha_{u}^{*}+\beta_{u}^{*}=1\right\}$ and recall $\bar{u}$ is the unique terminal with $\beta_{\bar{u}}^{*}=1 ; \bar{u}$ is clearly a member of $K$. At $\left(\alpha^{*}, \beta^{*}, \lambda^{*}\right)$, we evaluate the objective and collect like terms to get value

$$
\begin{aligned}
\sum_{U \in \mathcal{U}_{v}} z_{U} \rho(U \cap K)+\sum_{u \in K \backslash \bar{u}}\left(c_{u v}-\sum_{U: u \in U} z_{U}\right)+c_{\bar{u} v} & =\sum_{u \in K} c_{u v}+\sum_{U \in \mathcal{U}_{v}} z_{U}(\rho(U \cap K)-|(K \backslash \bar{u}) \cap U|) \\
& =\sum_{u \in K} c_{u v}-\sum_{U \in \mathcal{U}_{v}: U \cap K \neq \varnothing, \bar{u} \notin U} z_{U}
\end{aligned}
$$

where the last equality follows by considering cases. Finally, combining the fact that $\sum_{u \in K} c_{u v} \geq$ $C_{K}$ (since these edges form one possible full component on terminal set $K$ ) together with (4) for the pair $(K, \bar{u})$, it follows that the LP's optimal value is non-negative as needed.

Lemma 3.8. The incidence matrix defined by (D2') and (D3) is totally unimodular.

Proof. The incidence matrix has $\left|\mathcal{U}_{v}\right|+1$ rows $\left(\left|\mathcal{U}_{v}\right|\right.$ corresponding to (D2') and one last row corresponding to (D3)) and $\left|\mathcal{U}_{v}\right|+2|\Gamma(v)|$ columns. Furthermore, the columns corresponding to $\alpha_{u}$ 's are same as those corresponding to $\beta_{u}$ 's, except for the last row, where there are 0's in the $\alpha$-columns and 1's in the $\beta$-columns.

To show that this matrix is totally unimodular we use Ghouila-Houri's characterization of total unimodularity (e.g. see [30, Thm. 19.3]): 
Theorem 6 (Ghouila-Houri 1962). A matrix is totally unimodular iff the following holds for every subset $\mathcal{R}$ of rows: we can assign weights $w_{r} \in\{-1,+1\}$ to each row $r \in \mathcal{R}$ such that $\sum_{r \in \mathcal{R}} w_{r} r$ is $a\{0, \pm 1\}$-vector.

Note that we can safely ignore the columns corresponding to variables $\lambda_{U}$ for sets $U \in \mathcal{U}_{v}$, since each of them contains a single 1 occurring in constraint (D2') for set $U$.

The row subset $\mathcal{R}$ corresponds to a subset of $\mathcal{U}_{v}$ - which we will denote $\mathcal{R} \cap \mathcal{U}_{v}$ - plus possibly the single row corresponding to (D3). Each row in $\mathcal{R} \cap \mathcal{U}_{v}$ has its values determined by the characteristic vector of $U \cap \Gamma(v)$. So long as any set appears more than once in $\{U \cap \Gamma(v) \mid U \in$ $\left.\mathcal{R} \cap \mathcal{U}_{v}\right\}$ we can assign one copy weight +1 and the other copy weight -1 ; these rows cancel out. Thus, henceforth we assume $\left\{U \cap \Gamma(v) \mid U \in \mathcal{R} \cap \mathcal{U}_{v}\right\}$ has no duplicate sets.

There is a standard representation of a laminar family as a forest of rooted trees, where there is a node corresponding to each set, with containment in the family corresponding to ancestry in the forest. Given the forest for the laminar family $\left\{U \cap \Gamma(v) \mid U \in \mathcal{R} \cap \mathcal{U}_{v}\right\}$, the assignment of weights to the rows of the matrix is as follows. Let the root nodes of all trees be at height 0 with height increasing as one goes to children nodes. Give weight -1 to rows corresponding to nodes at even height, and weight +1 to rows corresponding to nodes at odd height. If $\mathcal{R}$ contains the row corresponding to (D3), give it weight +1 .

Finally, let us argue that these weights have the needed property. Consider first a column corresponding to $\alpha_{u}$ for any $u$. The rows of $\mathcal{R}$ with 1 in this column form a path, from the largest set containing $u$ (which is a root node) to the smallest set containing $u$. The weighted sum in this column is an alternating sum $-1+1-1+1 \cdots$, which is either -1 or 0 , which is in $\{0, \pm 1\}$ as needed. Second, in a column for some $\beta_{u}$, if $\mathcal{R}$ doesn't contain (resp. contains) the row corresponding to (D3), the weighted sum is the same as for $\alpha_{u}$ (resp. plus 1); in either case its weighted sum is in $\{0, \pm 1\}$ as needed.

This finishes the proof of Lemma 3.5, and hence also that of Theorem 5 .

\section{Improved Integrality Gap Upper Bounds}

We first show the improved bound of $73 / 60$ for uniformly quasibipartite graphs. We then show the $(2 \sqrt{2}-1) \doteq 1.828$ upper bound on general graphs, which contains the main ideas, and then end by giving a $\sqrt{3} \doteq 1.729$ upper bound.

\subsection{Uniformly Quasibipartite Instances}

Uniformly quasibipartite instances of the Steiner tree problem are quasibipartite graphs where the cost of edges incident on a Steiner vertex are the same. They were first studied by Gröpl et al. [20], who gave a 73/60 factor approximation algorithm. In the following, we show that the cost of the returned tree is no more than than $\frac{73}{60} \mathrm{OPT}(\mathcal{P})$, which upper-bounds the integrality gap by $\frac{73}{60}$.

We start by describing the algorithm of Gröpl et al. [20] in terms of full components. A collection $\mathcal{K}^{\prime}$ of full components is acyclic if there is no list of $t>1$ distinct terminals and hyperedges in $\mathcal{K}^{\prime}$ of the form $r_{1} \in K_{1} \ni r_{2} \in K_{2} \cdots \ni r_{t} \in K_{t} \ni r_{1}$ - i.e. there are no hypercycles. 


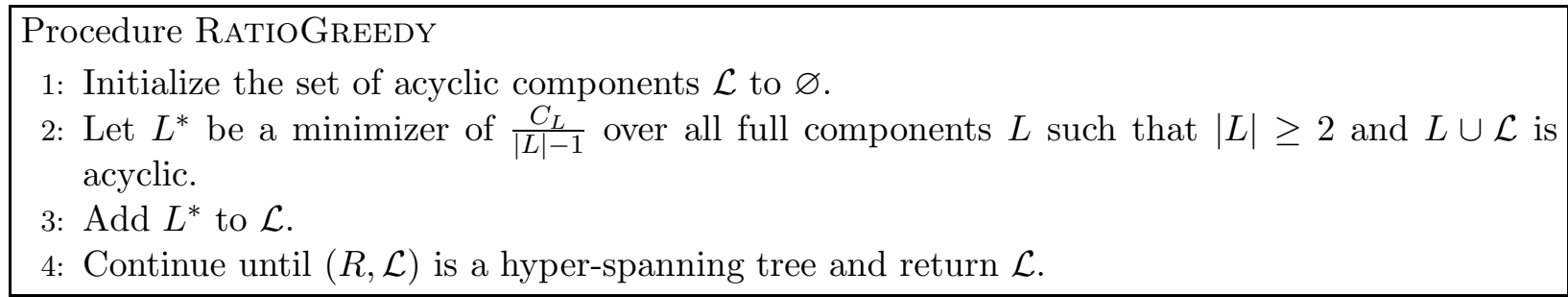

Theorem 7. On a uniformly quasibipartite instance RATIOGREEDY returns a Steiner tree of cost at most $\frac{73}{60} \mathrm{OPT}(\mathcal{P})$.

Proof. Let $t$ denote the number of iterations and $\mathcal{L}:=\left\{L_{1}, \ldots, L_{t}\right\}$ be the ordered sequence of full components obtained. We now define a dual solution to $\left(\mathcal{P}_{D}\right)$. Let $\pi(i)$ denote the partition induced by the connected components of $\left\{L_{1}, \ldots, L_{i}\right\}$. Let $\theta(i)$ denote $C_{L_{i}} /\left(\left|L_{i}\right|-1\right)$ and note that $\theta$ is nondecreasing. Define $\theta(0)=0$ for convenience. We define a dual solution $y$ with

$$
y_{\pi(i)}=\theta(i+1)-\theta(i)
$$

for $0 \leq i<t$, and all other coordinates of $y$ set to zero; $y$ is not generally feasible, but we will scale it down to make it so. By evaluating a telescoping sum, it is not hard to find that $\sum_{i} y_{\pi(i)}(r(\pi(i))-1)=C(\mathcal{L})$. In the rest of the proof we will show for any $K \in \mathcal{K}, \sum_{i} y_{\pi(i)} \mathrm{rc}_{K}^{\pi(i)} \leq$ $73 / 60 \cdot C_{K}$ - by scaling, this also proves that $\frac{60}{73} y$ is a feasible dual solution, and hence completes the proof.

Fix any $K \in \mathcal{K}$ and let $|K|=k$. Since the instance in question is uniformly quasi-bipartite, the full component $K$ is a star with a Steiner centre and edges of a fixed cost $c$ to each terminal in $K$. For $1 \leq i<k$, let $\tau(i)$ denote the last iteration $j$ in which $\operatorname{rc}_{K}^{\pi(j)} \geq k-i$. Let $K_{i}$ denote any subset of $K$ of size $k-i+1$ such that $K_{i}$ contains at most one element from each part of $\pi(\tau(i))$; i.e., $\left|K_{i}\right|=k-i+1$ and $\mathrm{rc}_{K_{i}}^{\pi(\tau(i))}=k-i$.

Our analysis hinges on the fact that $K_{i}$ was a valid choice for $L_{\tau(i)+1}$. More specifically, note that $\left\{L_{1}, \ldots, L_{\tau(i)}, K_{i}\right\}$ is acyclic, hence by the greedy nature of the algorithm, for any $1 \leq i<k$,

$$
\theta(\tau(i)+1)=C_{L_{\tau(i)+1}} /\left(\left|L_{\tau(i)+1}\right|-1\right) \leq C_{K_{i}} /\left(\left|K_{i}\right|-1\right) \leq \frac{c \cdot(k-i+1)}{k-i} .
$$

Moreover, using the definition of $\tau$ and telescoping we compute

$\sum_{\pi} y_{\pi} \mathrm{rc}_{K}^{\pi}=\sum_{i=0}^{t-1}(\theta(i+1)-\theta(i)) \mathrm{rc}_{K}^{\pi(i)}=\sum_{i=1}^{k-1} \theta(\tau(i)+1) \leq \sum_{i=1}^{k-1} \frac{c \cdot(k-i+1)}{k-i}=c \cdot(k-1+H(k-1))$,

where $H(\cdot)$ denotes the harmonic series. Finally, note that $(k-1+H(k-1)) \leq \frac{73}{60} k$ for all $k \geq 2$ (achieved at $k=5$ ). Therefore, $\frac{60}{73} y$ is a valid solution to $\left(\mathcal{P}_{D}\right)$.

\subsection{General graphs}

We start with a few definitions and notations in order to prove the $2 \sqrt{2}-1$ and $\sqrt{3}$ integrality gap bounds on $(\mathcal{P})$. Both results use similar algorithms, and the latter is a more complex version of the former. For conciseness we let a "graph" be a triple $G=(V, E, R)$ where $R \subset V$ are $G$ 's terminals. In the following, we let $\operatorname{mtst}(G ; c)$ denote the minimum terminal spanning tree, i.e. the minimum spanning tree of the terminal-induced subgraph $G[R]$ under edge-costs $c: E \rightarrow \mathbf{R}$. We will abuse notation and let $\operatorname{mtst}(G ; c)$ mean both the tree and its cost under $c$. 
When contracting an edge $u v$ in a graph, the new merged node resulting from contraction is defined to be a terminal iff at least one of $u$ or $v$ was a terminal; this is natural since a Steiner tree in the new graph is a minimal set of edges which, together with $u v$, connects all terminals in the old graph. Our algorithm performs contraction, which may introduce parallel edges, but one may delete all but the cheapest edge from each parallel class without affecting the analysis.

Our first algorithm proceeds in stages. In each stage we apply the operation $G \mapsto G / K$ which denotes contracting all edges in some full component $K$. To describe and analyze the algorithm we introduce some notation. For a minimum terminal spanning tree $T=\operatorname{mtst}(G ; c)$ define $\operatorname{drop}_{T}(K ; c):=c(T)-\operatorname{mtst}(G / K ; c)$. We also define gain $\operatorname{gin}_{T}(K ; c):=\operatorname{drop}_{T}(K)-c(K)$, where $c(K)$ is the cost of full component $K$. A tree $T$ is called gainless if for every full component $K$ we have $\operatorname{gain}_{T}(K ; c) \leq 0$. The following useful fact is implicit in [23] (see also Appendix B).

Theorem 8 (Implicit in [23]). If $\operatorname{mtst}(G ; c)$ is gainless, then $\operatorname{OPT}(\mathcal{P})$ equals the cost of $\operatorname{mtst}(G ; c)$.

We now give the first algorithm and its analysis, which uses a reduced cost trick introduced by Chakrabarty et al.[4].

Procedure Reduced OnE-PASs Heuristic
1: Define costs $c_{e}^{\prime}$ by $c_{e}^{\prime}:=c_{e} / \sqrt{2}$ for all terminal-terminal edges $e$, and $c_{e}^{\prime}=c_{e}$ for all other
edges. Let $G_{1}:=G, T_{i}:=\operatorname{mtst}\left(G_{i} ; c^{\prime}\right)$, and $i:=1$.
2: The algorithm considers the full components in any order. When we examine a full component
$\quad K$, if gain $\operatorname{t}_{i}\left(K ; c^{\prime}\right)>0$, let $K_{i}:=K, G_{i+1}:=G_{i} / K_{i}, T_{i+1}:=\operatorname{mtst}\left(G_{i+1} ; c^{\prime}\right)$, and $i:=i+1$.
3: Let $f$ be the final value of $i$. Return the tree $T_{a l g}:=T_{f} \cup \bigcup_{i=1}^{f-1} K_{i}$.

Note that the full components are scanned in any order and they are not examined a priori. Hence the algorithm works just as well if the full components arrive "online," which might be useful for some applications.

Theorem 9. $c\left(T_{\text {alg }}\right) \leq(2 \sqrt{2}-1) \operatorname{OPT}(\mathcal{P})$.

Proof. First we claim that gain $T_{T_{f}}\left(K ; c^{\prime}\right) \leq 0$ for all $K$. To see this there are two cases. If $K=K_{i}$ for some $i$, then we immediately see that $\operatorname{drop}_{T_{j}}(K)=0$ for all $j>i$ so $\operatorname{gain}_{T_{f}}(K)=-c(K) \leq 0$. Otherwise (if for all $\left.i, K \neq K_{i}\right) K$ had nonpositive gain when examined by the algorithm; and the well-known contraction lemma (e.g., see $[19, \S 1.5]$ ) immediately implies that gain $_{T_{i}}(K)$ is nonincreasing in $i$, so gain $T_{f}(K) \leq 0$.

By Theorem 8, $c^{\prime}\left(T_{f}\right)$ equals the value of $(\mathcal{P})$ on the graph $G_{f}$ with $\operatorname{costs} c^{\prime}$. Since $c^{\prime} \leq c$, and since at each step we only contract terminals, the value of this optimum must be at most OPT $(\mathcal{P})$. Using the fact that $c\left(T_{f}\right)=\sqrt{2} c^{\prime}\left(T_{f}\right)$, we get

$$
c\left(T_{f}\right)=\sqrt{2} c^{\prime}\left(T_{f}\right) \leq \sqrt{2} \mathrm{OPT}(\mathcal{P})
$$

Furthermore, for every $i$ we have gain $T_{T_{i}}\left(K_{i} ; c^{\prime}\right)>0$, that is, $\operatorname{drop}_{T_{i}}\left(K_{i} ; c^{\prime}\right)>c^{\prime}(K)=c(K)$. The equality follows since $K$ contains no terminal-terminal edges. However, $\operatorname{drop}_{T_{i}}\left(K_{i} ; c^{\prime}\right)=$ $\frac{1}{\sqrt{2}} \mathrm{drop}_{T_{i}}\left(K_{i} ; c\right)$ because all edges of $T_{i}$ are terminal-terminal. Thus, we get for every $i=1$ to $f, \operatorname{drop}_{T_{i}}\left(K_{i} ; c\right)>\sqrt{2} \cdot c\left(K_{i}\right)$.

Since $\operatorname{drop}_{T_{i}}\left(K_{i} ; c\right):=\operatorname{mtst}\left(G_{i} ; c\right)-\operatorname{mtst}\left(G_{i+1} ; c\right)$, we have

$$
\sum_{i=1}^{f-1} \operatorname{drop}_{T_{i}}\left(K_{i} ; c\right)=\operatorname{mtst}(G ; c)-c\left(T_{f}\right) .
$$


Thus, we have

$$
\sum_{i=1}^{f-1} c\left(K_{i}\right) \leq \frac{1}{\sqrt{2}} \sum_{i=1}^{f} \operatorname{drop}_{T_{i}}\left(K_{i} ; c\right)=\frac{1}{\sqrt{2}}\left(\operatorname{mtst}(G ; c)-c\left(T_{f}\right)\right) \leq \frac{1}{\sqrt{2}}\left(2 \mathrm{OPT}(\mathcal{P})-c\left(T_{f}\right)\right)
$$

where we use the fact that $\operatorname{mtst}(G, c)$ is at most twice $\mathrm{OPT}(\mathcal{P})^{6}$. Therefore

$$
c\left(T_{a l g}\right)=c\left(T_{f}\right)+\sum_{i=1}^{f-1} c\left(K_{i}\right) \leq\left(1-\frac{1}{\sqrt{2}}\right) c\left(T_{f}\right)+\sqrt{2} \operatorname{OPT}(\mathcal{P}) .
$$

Finally, using $c\left(T_{f}\right) \leq \sqrt{2} \mathrm{OPT}(\mathcal{P})$ from $(22)$, the proof of Theorem 9 is complete.

\subsubsection{Improving to $\sqrt{3}$}

To get the improved factor of $\sqrt{3}$, we use a more refined iterated contraction approach. The crucial new concept is that of the loss of a full component, introduced by Karpinski and Zelikovsky [22]. The intuition is as follows. In each iteration, the $(2 \sqrt{2}-1)$-factor algorithm contracts a full component $K$, and thus commits to include $K$ in the final solution; the new algorithm makes a smaller commitment, by contracting a subset of $K$ 's edges, which allows for a possibility of better recovery later.

Given a full component $K$ (viewed as a tree with leaf set $K$ and internal Steiner nodes), $\operatorname{loss}(K)$ is defined to be the minimum-cost subset of $E(K)$ such that $(V(K), \operatorname{loss}(K))$ has at least one terminal per connected component - i.e. the cheapest way in $K$ to connect each Steiner node to the terminal set. We also use $\operatorname{loss}(K)$ to denote the total cost of these edges. Note that no two terminals are connected by $\operatorname{loss}(K)$. A very useful theorem of Karpinski and Zelikovsky $[22]$ is that for any full component $K, \operatorname{loss}(K) \leq c(K) / 2$.

Now we have the ingredients to give our new algorithm. In the description below, $\alpha>1$ is a parameter (which will be set to $\sqrt{3}$ ). In each iteration, the algorithm contracts the loss of a single full component $K$ (we note it follows that the terminal set has constant size over all iterations).

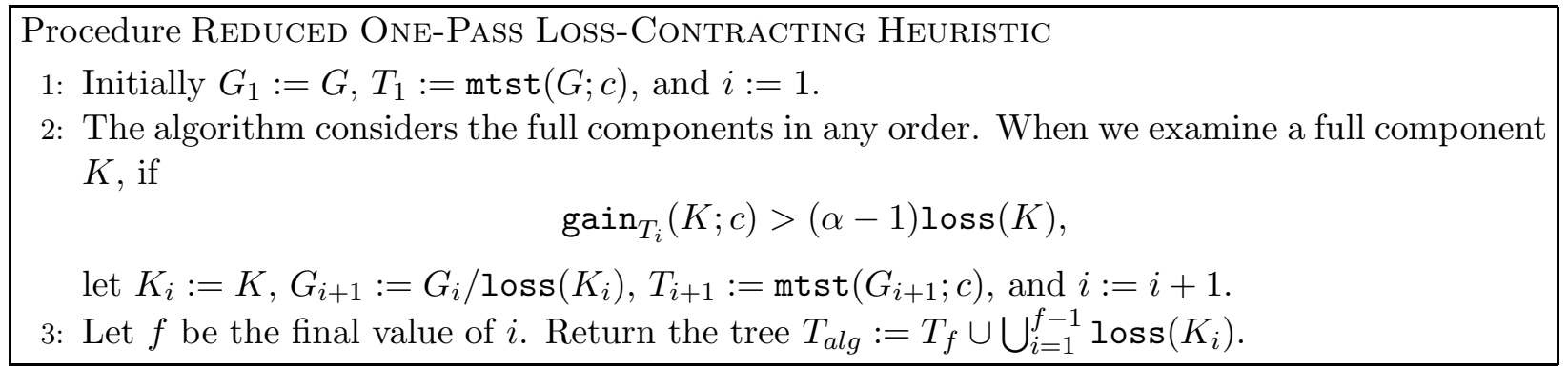

We now analyze the algorithm.

Claim 4.1. $c\left(T_{f}\right) \leq\left(\frac{1+\alpha}{2}\right) \operatorname{OPT}(\mathcal{P})$.

Proof. Using the contraction lemma again, $\operatorname{gain}_{T_{f}}(K ; c) \leq(\alpha-1) \operatorname{loss}(K)$ for all $K$, so

$$
\operatorname{drop}_{T_{f}}(K ; c) \leq c(K)+(\alpha-1) \operatorname{loss}(K)=c(K)+(\alpha-1) \operatorname{loss}(K) \leq\left(\frac{1+\alpha}{2}\right) c(K)
$$

\footnotetext{
${ }^{6}$ This follows using standard arguments, and can be seen, for instance, by applying Theorem 8 to the cost-function with all terminal-terminal costs divided by 2 , and using short-cutting.
} 
since $\operatorname{loss}(K) \leq c(K) / 2$.

To finish the proof of Claim 4.1, we proceed as in the proof of Equation (22). Define $c_{e}^{\prime}:=$ $c_{e} /\left(\frac{1+\alpha}{2}\right)$ for all edges $e$ which join two vertices of the original terminal set $R$, and $c_{e}^{\prime}=c_{e}$ for all other edges. Note that (23) implies that $T_{f}$ is gainless with respect to $c^{\prime}$. Thus, by Theorem 8 , the value of $\operatorname{LP}(\mathcal{P})$ on $\left(G_{f}, c^{\prime}\right)$ equals $c^{\prime}\left(T_{f}\right)$. Since we only reduce costs (as $\left.\alpha \geq 1\right)$, this optimum is no more than the original $\operatorname{OPT}(\mathcal{P})$ giving us $c^{\prime}\left(T_{f}\right) \leq \operatorname{OPT}(\mathcal{P})$. Now using the definition of $c^{\prime}$, the proof of the claim is complete.

Claim 4.2. For any $i \geq 1$, we have $c\left(T_{i}\right)-c\left(T_{i+1}\right) \geq \operatorname{gain}_{T_{i}}\left(K_{i} ; c\right)+\operatorname{loss}\left(K_{i}\right)$.

Proof. Recall that $T_{i+1}$ is a minimum terminal spanning tree of $G_{i+1}$ under $c$. Consider the following other terminal spanning tree $T$ of $G_{i+1}$ : take $T$ to be the union of $K_{i} / \operatorname{loss}\left(K_{i}\right)$ with mtst $\left(G_{i} / K_{i} ; c\right)$. Hence $c\left(T_{i+1}\right) \leq c(T)=\operatorname{mtst}\left(G_{i} / K_{i} ; c\right)+c\left(K_{i}\right)-\operatorname{loss}\left(K_{i}\right)$. Rearranging, and using the definition of gain, we obtain:

$$
c\left(T_{i}\right)-c\left(T_{i+1}\right) \geq c\left(T_{i}\right)-\operatorname{mtst}\left(G_{i} / K_{i} ; c\right)-c\left(K_{i}\right)+\operatorname{loss}\left(K_{i}\right)=\operatorname{gain}_{T_{i}}\left(K_{i} ; c\right)+\operatorname{loss}\left(K_{i}\right),
$$

and this completes the proof.

Now we are ready to prove the integrality gap upper bound of $\sqrt{3}$.

Theorem 10. $c\left(T_{\text {alg }}\right) \leq \sqrt{3} \mathrm{OPT}(\mathcal{P})$.

Proof. By the algorithm, we have for all $i$ that gain $T_{i}\left(K_{i}\right) \geq(\alpha-1) \operatorname{loss}\left(K_{i}\right)$, and thus gain $T_{i}\left(K_{i} ; c\right)+$ $\operatorname{loss}\left(K_{i}\right) \geq \alpha \operatorname{loss}\left(K_{i}\right)$. Thus, from Claim 4.2, we get

$$
\sum_{i=1}^{f-1} \operatorname{loss}\left(K_{i}\right) \leq \frac{1}{\alpha} \sum_{i=1}^{f-1}\left(c\left(T_{i}\right)-c\left(T_{i+1}\right)\right)
$$

The right-hand sum telescopes to give us $c\left(T_{1}\right)-c\left(T_{f}\right)=\operatorname{mtst}(G ; c)-c\left(T_{f}\right)$. Thus,

$$
\begin{aligned}
c\left(T_{a l g}\right) & =c\left(T_{f}\right)+\sum_{i=1}^{f-1} \operatorname{loss}\left(K_{i}\right) \leq c\left(T_{f}\right)+\frac{1}{\alpha}\left(\operatorname{mtst}(G ; c)-c\left(T_{f}\right)\right)=\frac{1}{\alpha} \operatorname{mtst}(G ; c)+\frac{\alpha-1}{\alpha} c\left(T_{f}\right) \\
& \leq\left(\frac{2}{\alpha}+\frac{(\alpha-1)(1+\alpha)}{2 \alpha}\right) \operatorname{OPT}(\mathcal{P})=\left(\frac{\alpha^{2}+3}{2 \alpha}\right) \operatorname{OPT}(\mathcal{P})
\end{aligned}
$$

which follows from $\operatorname{mtst}(G ; c) \leq 2 \mathrm{OPT}(\mathcal{P})$ and Claim 4.1. Setting $\alpha=\sqrt{3}$, the proof of the theorem is complete.

\section{Conclusion}

In this paper we looked at several hypergraphic LP relaxations for the Steiner tree problem, and showed they all have the same objective value. Furthermore, we noted some connections to the bidirected cut relaxation for Steiner trees: although hypergraphic relaxations are stronger than the bidirected cut relaxation in general, in quasibipartite graphs all these relaxations are equivalent. We obtained structural results about the hypergraphic relaxations showing that basic feasible solutions have sparse support. We also showed improved upper bounds on the integrality gaps on the hypergraphic relaxations via simple algorithms.

Reiterating the comments in Section 1.2.3, the hypergraphic LPs are powerful (e.g. as evidenced by Byrka et al. [3]) but may not be manageable for computational implementation. Some interesting 
areas for future work include: non-ellipsoid-based algorithms to solve the hypergraphic LPs in the $r$-restricted setting; resolving the complexity of optimizing them in the unrestricted setting; and directly using the bidirected cut relaxation to achieve good results (e.g. in quasi-bipartite instances).

\section{References}

[1] Y. P. Aneja. An integer linear programming approach to the Steiner problem in graphs. Networks, 10:167-178, 1980.

[2] A. Borchers and D. Du. The k-Steiner ratio in graphs. SIAM J. Comput., 26(3):857-869, 1997.

[3] J. Byrka, F. Grandoni, T. Rothvoß, and L. Sanità. An improved LP-based approximation for Steiner tree. To appear in Proc. 42nd STOC, 2010.

[4] D. Chakrabarty, N. R. Devanur, and V. V. Vazirani. New geometry-inspired relaxations and algorithms for the metric Steiner tree problem. In IPCO, pages 344-358, 2008.

[5] M. Chlebík and J. Chlebíková. Approximation hardness of the Steiner tree problem on graphs. In Proceedings, Scandinavian Workshop on Algorithm Theory, pages 170-179, 2002.

[6] S. Chopra. On the spanning tree polyhedron. Operations Research Letters, 8:25-29, 1989.

[7] S. Chopra and M. R. Rao. The Steiner tree problem 1: Formulations, compositions, and extension of facets. Mathematical Programming, 64:209-229, 1994.

[8] S. Chopra and M. R. Rao. The Steiner tree problem 2: Properties and classes of facets. Mathematical Programming, 64:231-246, 1994.

[9] G. Cornuéjols, D. Naddef, and J. Fonlupt. The traveling salesman problem on a graph and some related integer polyhedra. Math. Programming, 33:1-27, 1985.

[10] M. Didi Biha, H. Kerivin, and A. R. Mahjoub. Steiner trees and polyhedra. Discrete Applied Mathematics, 112(1-3):101-120, 2001.

[11] J. Edmonds. Optimum branchings. Journal of Research of the National Bureau of Standards B, 71B:233-240, 1967.

[12] J. Edmonds. Matroids and the greedy algorithm. Math. Programming, 1:127-136, 1971.

[13] J. Edmonds and R. Giles. A min-max relation for submodular functions on graphs. Annals of Discrete Mathematics, 1:185-204, 1977.

[14] A. Frank, T. Király, and Z. Király. On the orientation of graphs and hypergraphs. Discrete Appl. Math., 131(2):385-400, 2003.

[15] D. R. Fulkerson. Blocking and anti-blocking pairs of polyhedra. Math. Programming, 1:168194, 1971.

[16] D. Gijswijt and G. Pap. An algorithm for weighted fractional matroid matching. arXiv:0806.1818, 2008. 
[17] M. X. Goemans. The Steiner tree polytope and related polyhedra. Math. Program., 63(2):157$182,1994$.

[18] M. X. Goemans and Y. Myung. A catalog of Steiner tree formulations. Networks, 23:19-28, 1993.

[19] C. Gröpl, S. Hougardy, T. Nierhoff, and H. J. Prömel. Approximation algorithms for the Steiner tree problem in graphs. In X. Cheng and D. Du, editors, Steiner trees in industries, pages 235-279. Kluwer Academic Publishers, Norvell, Massachusetts, 2001.

[20] C. Gröpl, S. Hougardy, T. Nierhoff, and H. J. Prömel. Steiner trees in uniformly quasibipartite graphs. Inform. Process. Lett., 83(4):195-200, 2002. Preliminary version appeared as a Technical Report at TU Berlin, 2001.

[21] K. Jain. A factor 2 approximation algorithm for the generalized Steiner network problem. Combinatorica, 21(1):39-60, 2001. Preliminary version appeared in Proc. 39th FOCS, pages 448-457, 1998.

[22] M. Karpinski and A. Zelikovsky. New approximation algorithms for the Steiner tree problems. J. Combinatorial Optimization, 1(1):47-65, 1997.

[23] J. Könemann, D. Pritchard, and K. Tan. A partition-based relaxation for Steiner trees. Math. Programming, 2009. [In press].

[24] T. Polzin. Algorithms for the Steiner Problem in Networks. PhD thesis, Universität des Saarlandes, February 2003.

[25] T. Polzin and S. Vahdati Daneshmand. A comparison of Steiner tree relaxations. Discrete Applied Mathematics, 112(1-3):241-261, 2001. Preliminary version appeared at COS 1998.

[26] T. Polzin and S. Vahdati Daneshmand. On Steiner trees and minimum spanning trees in hypergraphs. Oper. Res. Lett., 31(1):12-20, 2003.

[27] D. Pritchard. Linear Programming Tools \& Approximation Algorithms for Combinatorial Optimization. PhD thesis, University of Waterloo, 2009.

[28] S. Rajagopalan and V. V. Vazirani. On the bidirected cut relaxation for the metric Steiner tree problem. In Proceedings, ACM-SIAM Symposium on Discrete Algorithms, pages 742-751, 1999.

[29] G. Robins and A. Zelikovsky. Tighter bounds for graph Steiner tree approximation. SIAM J. Discrete Math., 19(1):122-134, 2005. Preliminary version appeared as "Improved Steiner tree approximation in graph" at SODA 2000.

[30] A. Schrijver. Theory of Linear and Integer Programming. Wiley, Chichester, 1986.

[31] M. Singh and L. C. Lau. Approximating minimum bounded degree spanning trees to within one of optimal. In Proc. 39th STOC, pages 661-670, 2007.

[32] R. P. Stanley. Enumerative Combinatorics, volume 1. Wadsworth \& Brooks/Cole, 1986.

[33] J. H. V. Vate. Fractional matroid matchings. J. Comb. Theory, Ser. B, 55(1):133-145, 1992. 
[34] V. Vazirani. Recent results on approximating the Steiner tree problem and its generalizations. Theoret. Comput. Sci., 235(1):205-216, 2000.

[35] D. Warme. Spanning Trees in Hypergraphs with Applications to Steiner Trees. PhD thesis, University of Virginia, 1998.

[36] R. T. Wong. A dual ascent approach for Steiner tree problems on a directed graph. Math. Programming, 28:271-287, 1984.

\section{A Directed Hypergraph LP Relaxation}

Theorem 11. For any Steiner tree instance, $\mathrm{OPT}(\mathcal{P})=\operatorname{OPT}(\mathcal{D})$.

Proof. First, we show $\operatorname{OPT}(\mathcal{P}) \leq \operatorname{OPT}(\mathcal{D})$. Consider a feasible solution $x$ to $(\mathcal{D})$, and define a solution $x^{\prime}$ to $(\mathcal{P})$ by $x_{K}^{\prime}=\sum_{i \in K} x_{K^{i}}$; informally, $x^{\prime}$ is obtained from $x$ by ignoring the orientation of the hyperedges. Clearly $x^{\prime}$ and $x$ have the same objective value. Further, $x^{\prime}$ is feasible for $(\mathcal{P})$; to see this, for any partition $\pi$, note that $(5)$ is implied by the sum of constraints (3) over $U$ set to those parts of $\pi$ not containing the root - any orientation of a full component with rank contribution $t$ must leave at least $t$ parts.

To obtain the reverse direction $\operatorname{OPT}(\mathcal{D}) \leq \mathrm{OPT}(\mathcal{P})$, we use a similar strategy. We require some notation and a hypergraph orientation theorem of Frank et al. [14]. For any $U \subset R$ we say that a directed hyperedge $K^{i}$ lies in $\Delta^{\mathrm{in}}(U)$ if $i \in U$ and $K \backslash U \neq \varnothing$, i.e. if $K^{i} \in \Delta^{\text {out }}(R \backslash U)$. Two subsets $U$ and $W$ of $R$ are called crossing if all four sets $U \backslash W, W \backslash U, U \cap W$, and $R \backslash(U \cup W)$ are non-empty. A set-function $p: 2^{R} \rightarrow \mathbb{Z}$ is a crossing supermodular function if

$$
p(U)+p(W) \leq p(U \cap W)+p(U \cup W)
$$

for all crossing sets $U$ and $W$. A directed hypergraph is said to cover $p$ if $\left|\Delta^{\text {in }}(U)\right| \geq p(U)$ for all $U \subset R$. Here is the needed result.

Theorem 12 (Frank, Király \& Király [14]). Given a hypergraph $H=(R, \mathcal{X})$, and a crossing supermodular function $p$, the hypergraph has an orientation covering $p$ if and only if for every partition $\pi$ of $R$,

(a) $\sum_{K \in \mathcal{X}} \min \left\{1, \mathrm{rc}_{K}^{\pi}\right\} \geq \sum_{\pi_{i} \in \pi} p\left(\pi_{i}\right)$, and, (b) $\sum_{K \in \mathcal{X}} \mathrm{rc}_{K}^{\pi} \geq \sum_{\pi_{i} \in \pi} p\left(R \backslash \pi_{i}\right)$.

We will show every rational solution $x$ to $(\mathcal{P})$ can be fractionally oriented to get a feasible solution for $(\mathcal{D})$, which will complete the proof of Theorem 11. Let $M$ be the smallest integer such that the vector $M x$ is integral. Let $\mathcal{X}$ be a multi-set of hyperedges which contains $M x_{K}$ copies of each $K$. Define the function $p$ by $p(U)=M$ if $r \in U \neq R$, and $p(U)=0$ otherwise; i.e. $p(U)=M$ iff $R \backslash U$ is valid.

Claim A.1. $H=(R, \mathcal{X})$ satisfies conditions (a) and (b).

Proof. Note $\sum_{\pi_{i} \in \pi} p\left(R \backslash \pi_{i}\right)=M(r(\pi)-1)$ since all parts of $\pi$ are valid except the part containing the root $r$. Thus condition (b), upon scaling by $\frac{1}{M}$, is a restatement of constraint (5), which holds since $x$ is feasible for $(\mathcal{P})$.

For this $p$, condition (a) follows from (b) in the following sense. Fix a partition $\pi$, and let $\pi_{1}$ be the part of $\pi$ containing $r$. If $\pi_{1}=R$ then (a) is vacuously true, so assume $\pi_{1} \neq R$. Let $\sigma$ be the rank-2 partition $\left\{\pi_{1}, R \backslash \pi_{1}\right\}$. Then it is easy to check that $\min \left\{1, \mathrm{rc}_{K}^{\pi}\right\} \geq \mathrm{rc}_{K}^{\sigma}$ for all $K$, and consequently $\sum_{K \in \mathcal{X}} \min \left\{1, \mathrm{rc}_{K}^{\pi}\right\} \geq \sum_{K \in \mathcal{X}} \mathrm{rc}_{K}^{\sigma}$ and $\sum_{\pi_{i} \in \sigma} p\left(R \backslash \pi_{i}\right)=M=\sum_{\pi_{i} \in \pi} p\left(\pi_{i}\right)$. Thus, (a) for $\pi$ follows from (b) for $\sigma$. 
It is not hard to check that $p$ is crossing supermodular. Now using Theorem 12, take an orientation of $\mathcal{X}$ that covers $p$.

For each $K \in \mathcal{K}$ and each $i \in K$, let $n_{K^{i}}$ denote the number of the $M x_{K}$ copies of $K$ that are oriented as $K^{i}$, i.e. directed towards $i$. So, $\sum_{i \in K} n_{K^{i}}=M x_{K}$. Let $x_{K^{i}}^{\prime}:=\frac{n_{K^{i}}}{M}$ for all $K^{i}$. Hence $\sum_{i} x_{K^{i}}^{\prime}=x_{K}$ and $x^{\prime}$ has the same objective value as $x$.

To complete the proof, we show $x^{\prime}$ is feasible for $(\mathcal{D})$. Fix a valid subset $U$ and consider condition (3) for a valid set $U$. Note that $p(R \backslash U)=M$. Therefore, since the orientation covers $p$, we get

$$
\sum_{K^{i} \in \Delta^{\text {out }}(U)} x_{K^{i}}^{\prime}=\frac{1}{M} \sum_{K^{i} \in \Delta^{\text {out }}(U)} n_{K^{i}}=\frac{1}{M} \sum_{K^{i} \in \Delta^{\operatorname{in}}(R \backslash U)} n_{K^{i}} \geq \frac{1}{M} p(R \backslash U)=\frac{1}{M} M=1
$$

as needed.

\section{B Gainless MSTs and Hypergraphic Relaxations}

Theorem 8 (Implicit in [23]). If the MST induced by the terminals is gainless, then OPT $(\mathcal{P})$ equals the cost of that MST.

Proof. Let $\Pi$ be the set of all partitions of the terminal set. As before, we let $r(\pi)$ be the rank of a partition $\pi \in \Pi$, and we use $E_{\pi}$ for the set of edges in our graph that cross the partition; i.e., $E_{\pi}$ contains all edges whose endpoints lie in different parts of $\pi$. Fulkerson's [15] formulation of the spanning tree polyhedron and its dual are as follows.

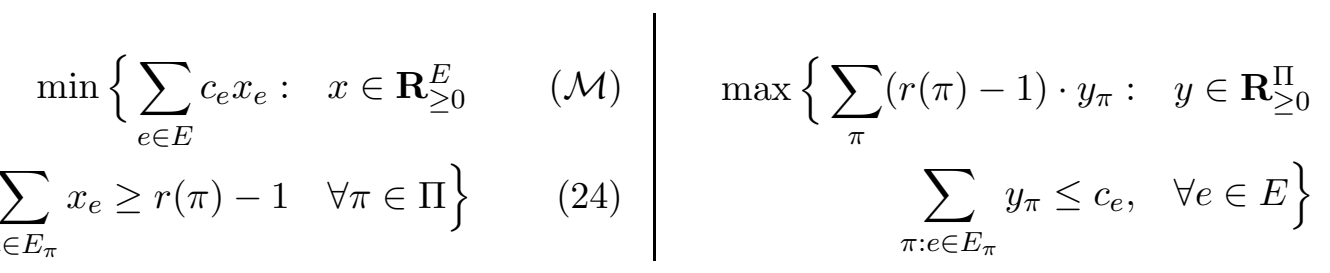

The high-level overview of the proof is as follows. We first give a brief sketch of a folklore primaldual interpretation of Kruskal's minimum-spanning tree algorithm with respect to Fulkerson's LP (for more information see, e.g., [23]). Running Kruskal's algorithm on the terminal set then returns a minimum spanning tree $T$ and a feasible dual $y$ to Equation $\left(\mathcal{M}_{D}\right)$ such that

$$
c(T)=\sum_{\pi}(r(\pi)-1) y_{\pi} .
$$

The final step will be to show that, if the returned MST is gainless, then the spanning tree dual $y$ is feasible for $\left(\mathcal{P}_{D}\right)$, and its value is $c(T)$ as well. Weak duality and the fact that the optimal value of $(\mathcal{P})$ is at most $c(T)$ imply the theorem.

Kruskal's algorithm can be viewed as a process over time. For each time $\tau \geq 0$, the algorithm keeps a forest $T^{\tau}$, and a feasible dual solution $y^{\tau}$; initially $T^{0}=(V, \varnothing)$ and $y^{0}=0$. Let $\pi^{\tau}$ be the partition induced by the connected components of $T^{\tau}$. If $T^{\tau}$ is not a spanning tree, Kruskal's algorithm grows the dual variable $y_{\pi^{\tau}}$ corresponding to the current partition until constraint Equation $\left(\mathcal{M}_{D}\right)$ e: for some edge $e$ prevents any further increase. The algorithm then adds $e$ to the partial tree and continues. The algorithm stops at the first time $\tau^{*}$ where $T^{\tau^{*}}$ is a spanning tree.

Let $T$ be the gainless spanning tree returned by Kruskal, and let $y$ be the corresponding dual. We claim that $y$ is feasible for $\left(\mathcal{P}_{D}\right)$. To see this, consider a full component $K$. Clearly, the rank contribution $\mathrm{rc}_{K}^{\pi^{0}}$ of $K$ to the initial partition $\pi^{0}$ is $|K|-1$; similarly, the final rank contribution 
$\mathrm{rc}_{K}^{\pi^{\tau^{*}}}$ is 0 . Every edge that is added during the algorithm's run either leaves the rank contribution of $K$ unchanged, or it decreases it by 1 . Let $e_{1}, \ldots, e_{|K|-1}$ be the edges of the final tree $T$ whose addition to $T$ decreases $K$ 's rank contribution. Also let

$$
0 \leq \tau_{1} \leq \tau_{2} \leq \ldots \leq \tau_{|K|-1} \leq \tau^{*}
$$

be the times where these edges are added. Note that, by definition, we must have $c_{e_{i}}=\tau_{i}$ for all $i$. We therefore have

$$
\sum_{i=1}^{|K|-1} c_{e_{i}}=\sum_{i=1}^{|K|-1} \tau_{i} .
$$

The right-hand side of this equality is easily checked to be equal to

$$
\int_{0}^{\tau^{*}} \mathrm{rc}_{K}^{\pi^{\tau}} d \tau
$$

which in turn is equal to $\sum_{\pi} \mathrm{rc}_{K}^{\pi} y_{\pi}$, by the definition of Kruskal's algorithm. It is not hard to see

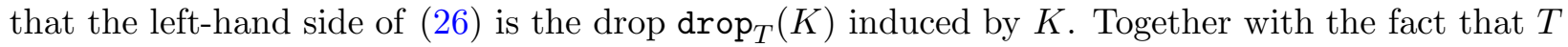
is gainless, we obtain

$$
c_{K} \geq \operatorname{drop}_{T}(K)=\sum_{\pi} \mathrm{rc}_{K}^{\pi} y_{\pi} .
$$

Now observe that the right-hand side of this equation is the left-hand side of (6). It follows that $y$ is feasible for $\left(\mathcal{P}_{D}\right)$. 